\title{
Fluid Approximation of Closed Queueing Networks with Discriminatory Processor Sharing
}

\author{
Lulai Zhu ${ }^{\mathrm{a}}$, Giuliano Casale ${ }^{\mathrm{a}}$, Iker Perez ${ }^{\mathrm{b}}$, \\ ${ }^{a}$ Department of Computing, Imperial College London, UK \\ ${ }^{b}$ School of Mathematical Sciences, University of Nottingham, UK
}

\begin{abstract}
As a multi-class variant of the classical egalitarian processor-sharing (EPS) discipline, discriminatory processor sharing (DPS) provides a suitable paradigm to model systems where share exists to control the service access of heterogeneous jobs. Although DPS is a more fine-grained scheduling discipline than EPS, the behavior of closed queueing networks (QNs) with DPS remains poorly understood. We propose in this paper a fluid approach to transient and steady-state analysis of closed QNs comprising DPS stations. Our reference model features an arbitrary routing topology, phase-type service time distributions and class switching. The proposed approach has been validated against simulation for both transient and steady-state analysis. We also apply the proposed transient analysis for approximating response time distributions at individual stations and for the entire system, showing its accuracy compared to prior art in handling non-exponential distributions.
\end{abstract}

Keywords: fluid approximation; closed queueing networks; discriminatory processor sharing; transient and steady-state analysis; response time distributions

\section{Introduction}

The vast majority of general-purpose operating systems, such as Linux, schedule multiple tasks on a single processor in a time-sharing manner such that each task in turn gains a time slice of the processor per cycle. Similarly, in packet-switched communication networks the bandwidth of a link 5 is distributed among all flows passing through it, and accordingly the rate reserved for each flow varies with respect to the total number of flows on the link. A number of scheduling policies are available in queueing theory to abstract these resource allocation mechanisms. The best-known one is perhaps the egalitarian processor-sharing (EPS) discipline, which assumes that all the jobs equally share the service capacity of the system. In spite of its mathematical tractability, and although each class can feature a different service time, the EPS model is unable to differentiate

Email addresses: lulai.zhu15@imperial.ac.uk (Lulai Zhu), g.casale@imperial.ac.uk (Giuliano Casale), Iker.Perez@nottingham.ac.uk (Iker Perez) 
the capacity share among the classes. Two multi-class variants of EPS have thus been developed, namely generalized processor sharing (GPS) [1, 2] and discriminatory processor sharing (DPS) [3]. The GPS discipline guarantees each running class a minimum portion of the service capacity. As for DPS, the portion of the service capacity for a class is not guaranteed, but rather depends on the number of jobs in each class residing at the resource.

The DPS discipline is widely applied in performance modeling of computer and communication systems. It naturally idealizes the weighted round-robin algorithm, which is often implemented in time-sharing operating systems to schedule prioritized tasks [4. Such an idealization is reasonable if the time slice gained by each task per cycle is very small compared to its processing time and if the temporal overhead spent in switching between two consecutive tasks is negligible.

Modern communication networks also adapt the quality of service for different groups of customers. This feature is well captured by the DPS discipline. For example, a single DPS queueing system has been used in [5] to evaluate the flow-level performance of unequal bandwidth sharing in the Internet. More applications of DPS can be found in [6], [7, 8], [9] and [10. Additionally, we mention [11, [12] and [13] as surveys on the DPS discipline overviewing relevant applications and results in the literature.

Queueing models with DPS lack in the general case a product-form solution. That is, the steady-state probabilities are not known to be decomposable into simple products, as instead available for EPS through the BCMP theorem [14]. Partly motivated by the need for insights into the performance of modern communication networks, much effort has then been put into understanding the behavior of single DPS queueing systems. There is however a shortage of results on the DPS discipline for closed queueing networks (QNs), which are useful to model, for example, complex software systems with admission control or threading limits on the interactions between separate software layers [15]. To address this limitation, we propose a fluid approach to analyzing the transient and steady-state behavior of closed QNs that comprise delay (i.e., infinite server) and DPS stations. Our reference model accommodates for an arbitrary routing topology among the stations. It also assumes the service time distributions for each class to be of phase type and allows a job to switch from one class to another upon service completion at a station.

The proposed approach is based on Kurtz's mean-field approximation [16], whereby a closed QN that can be casted as density-dependent Markov process is approximated by a system of ordinary differential equations (ODEs) where the discrete population of jobs is approximated as a continuous fluid that visits the resources. A specific problem in fluid approximation of closed QNs with DPS is that the resulting ODE system is discontinuous when a DPS station is empty. To address this technical issue, we modify the considered model by the notion of self-looping jobs [17, 45 i.e., jobs that permanently loop at a queueing station, consuming a fraction of the service capacity. Self-looping jobs have recently proven to be helpful in simplifying analysis of product-form closed 
QNs [18]. Unlike prior work, we show how this notion is applicable to closed QNs with DPS, for which a product-form solution is not known. Such a modification results in a stable and accurate fluid approximation to the original model under the condition that DPS stations are highly utilized.

50 and on typical examples for transient analysis.

One key benefit of transient analysis, which is simple to carry out from the fluid ODE results, is that they can be modified to compute the response time distribution of a tagged job, either visiting an individual station or in its entire visit to the system. To leverage this, along with the mating response time distributions at each station and further extend it to enable approximation of system-wide response time distributions with respect to a delay station. These methods are defined to natively cope with class switching through the concept of chains [19. They normally outperform prior art [20] with a significantly better accuracy for non-exponential service time distributions of phase-type.

The rest of the paper is organized as follows. Section 2 outlines related work on the DPS discipline. Our reference model is defined in Section 3, whereas the fluid approach is formulated in Section 4. The methods for approximating the response time distributions are described in Section 5. Section 6 discusses the settings and results of the validation. Section 7 is dedicated to of our results.

\section{Related Work}

DPS was initially suggested by Kleinrock to model time-sharing computer systems with priority groups [3]. Under the DPS discipline, each class- $k$ job in the system is assigned a non-negative weight $w_{k}$, and the service capacity is divided among all the jobs in proportion to their assigned weights. If there are $n_{l}(t)$ class- $l$ jobs in the system at time $t$, each class- $k$ job receives an instantaneous fraction $w_{k} / \sum_{l} w_{l} n_{l}(t)$ of the service capacity. By adjusting the class-dependent weights, one can therefore effectively control the actual completion rates of jobs in different classes. EPS may also be seen as a special case of DPS when all the jobs have the same weight.

After the work of Kleinrock, the research community mainly focused on DPS queueing systems with Poisson arrivals. Fayolle et al. 21] obtained the conditional average response times as the solution to a system of integro-differential equations. In the case of exponential service time distributions, this yields rather simple formulae, and the unconditional average response times satisfy a linear equation system. Rege and Sengupta [22] proved a decomposition theorem for the conditional response time distribution of a tagged job. Specifically, it was shown in [22] that the response time distribution of a job that sees $n$ jobs in the system upon arrival can be 
decomposed into $n+1$ independent components given by a system of non-linear integral equations. For exponentially distributed service times, Rege and Sengupta [23] also found the second-order moments of the joint queue length distribution as the solutions to linear simultaneous equations and proved a heavy-traffic limit theorem. These results were further extended to phase-type service time distributions by van Kessel et al. [24]. Avrachenkov et al. 11] showed that in a stable system, the mean queue lengths are finite irrespective of the higher-order moments of the service time distributions. Moreover, they derived a closed-form expression for the asymptote of the conditional average response time for each class, assuming that the service time distributions have finite second-order moments. Borst et al. 25] explored the asymptotic bounds of the response time distributions for heavy-tailed service time distributions. It was proven in [25] that under certain assumptions, the service time and response time distributions for a given class exhibit similar tail behavior independently of the service weights. Notably in the case of exponentially distributed service times, Kim and Kim [26] obtained a system of partial differential equations for the mixed transforms of the response time distribution for each class and the joint queue length distribution. They found by applying this equation system that the second-order moments of the response time distributions satisfy linear simultaneous equations. Based on Kim and Kim's work, a moment-generating algorithm for iteratively computing the higher-order moments of the response time distributions was devised by Chis and Harrison [27. Izagirre et al. 28] meanwhile derived a closed-form approximation to the average response time for each class, which is applicable for general service time distributions with finite second-order moments. This approximation becomes exact in some scenarios and is uniformly bounded by the second-order moments of the service time distributions.

Publications on closed queueing networks with DPS are instead limited in number. Mitra and Weiss 29] studied a model comprised of a delay and a DPS station where the think and service times are exponentially distributed. They obtained a system of ordinary differential equations for the transient mean queue lengths through asymptotic analysis. Under the heavy-traffic assumption, they gave explicit expressions for the steady-state values and a simple procedure for determining time constants that govern the approach to the steady state. Morrison [30] reviewed the same model but assumed moderately heavy traffic. He found two-term asymptotic approximations to the mean queue length and the average response time for each class as well as the leading term in the asymptotic approximation to the joint queue length distribution, which is a zero-mean multivariate Gaussian distribution.

\section{Model Definition}

We consider a closed QN that consists of $M$ stations and $K$ classes. There is a total population of $N$ jobs circulating among the stations, each of which belongs to one of the classes at any point 
in time. The service time of a class- $k$ job at station $i$ follows a phase-type distribution composed of $R_{i, k}$ phases. Each phase $r$ represents an exponentially distributed amount of service time with rate $\mu_{i, k, r}$ and is associated with a probability $\alpha_{i, k, r}$ of being chosen as the initial one. On completion of its service in phase $r$, the job shifts to phase $s$ with probability $P_{i, k,(r, s)}$ or alternatively ends in phase $r$ with probability $\beta_{i, k, r}=1-\sum_{s=1}^{R_{i, k}} P_{i, k,(r, s)}$. The type of a station can be either delay (i.e., infinite server) or DPS. We denote by $\mathcal{D}$ and $\mathcal{Q}$ the sets of delay and DPS stations respectively. A delay station is equipped with an infinite number of servers, thus allocating a dedicated server to each incoming job. In our case, a DPS station refers to a single-server queueing station that implements the DPS discipline. Each class- $k$ job at a DPS station $i$ is assigned a non-negative weight $w_{i, k}$ and receives a fraction $w_{i, k} / \sum_{l=1}^{K} w_{i, l} \sum_{s=1}^{R_{i, l}} n_{i, l, s}(t)$ of the server at time $t$, where $n_{i, l, s}(t)$ is the instantaneous number of class- $l$ jobs in phase $s$ at the station. Once the service of a class- $k$ job at station $i$ finishes, it switches to class $l$ and moves to station $j$ with probability $P_{(i, k),(j, l)}$. The routing probability matrix $\boldsymbol{P}=\left[P_{(i, k),(j, l)}\right] \in \mathbb{R}^{M K \times M K}$ is required to meet the assumption of a multi-chain QN (see Section 5.1). For convenience, we denote by $P_{(i, k, r),(j, l, s)}$ the probability of a class- $k$ job complete in phase $r$ at station $i$ proceeding to station $j$ as a class- $l$ job in phase $s$ :

$$
P_{(i, k, r),(j, l, s)}= \begin{cases}P_{i, k,(r, s)}+\beta_{i, k, r} P_{(i, k),(i, k)} \alpha_{i, k, s} & \text { if } i=j \wedge k=l, \\ \beta_{i, k, r} P_{(i, k),(j, l)} \alpha_{j, l, s} & \text { otherwise. }\end{cases}
$$

The former piece of 11 is due to the fact that when $i, j$ and $k, l$ index the same station and the same class respectively, the job may shift from phase $r$ to phase $s$ directly or otherwise end in phase $r$, loop back to the station and restart in phase $s$. Appendix A summarizes the main notation used throughout this paper.

\section{Fluid approximation}

Our reference model can be readily mapped onto a continuous-time Markov chain (CTMC) for which a steady-state probability vector uniquely exists. However, the state space of this CTMC is extremely large even for a medium-sized model instance. Fluid approximation treats the evolution of a discrete-state stochastic process in a continuous fashion, thus being an effective means to avoid the state space explosion [31]. Thanks to results obtained by Kurtz [16, the expected sample path of a model with a Markovian description can be approximated by the solution to a system of ODEs, provided that it satisfies a number of relatively mild technical conditions.

Featuring an arbitrary routing topology, the considered model may be instantiated as a simple closed QN where a delay station interacts with a DPS station. As shown in [29], the ODE system for such a model instance is discontinuous when the DPS station is empty, and consequently does not admit a unique solution. We identify this as a common problem in fluid approximation of 
closed QNs with DPS and overcome it by adding a self-looping job to each DPS station. Recall finishes [17. To apply this notion, we assign the self-looping job at a DPS station $i$ a service weight $w_{i, 0}$, which can be regarded as a function $g: \mathbb{R}^{K} \rightarrow \mathbb{R}$ of the local service weight vector $\boldsymbol{w}_{i}=\left(w_{i, k}\right) \in \mathbb{R}^{K}$ such that

$$
w_{i, 0}=\left\{\begin{array}{l}
g\left(\boldsymbol{w}_{i}\right) \geq 0, \\
g\left(\boldsymbol{w}_{i}\right)=a \quad \text { if } \forall k \in[1 \ldots K], w_{i, k}=a .
\end{array}\right.
$$

The former piece of $(2)$ is necessary for a valid service weight, while the latter piece is needed to

$\boldsymbol{x} \in \mathbb{R}^{R}$. It is straightforward that the above properties hold on the entire domain $\left\{\boldsymbol{x} \in \mathbb{R}^{R}\right\}$ in this case. Therefore, $f(\boldsymbol{x}, \boldsymbol{l})$ can be thought of as a function of the state argument $\boldsymbol{x}$ that is continuous, bounded and Lipschitz continuous on any non-empty compact and convex subset of its global domain $D=\left\{\boldsymbol{x} \in \mathbb{R}^{R} \mid \forall i \in \mathcal{Q}, \sum_{k^{\prime}=1}^{K} w_{i, k^{\prime}} \sum_{r^{\prime}=1}^{R_{i, k^{\prime}}} x_{i, k^{\prime}, r^{\prime}}+g\left(\boldsymbol{w}_{i}\right) \neq 0\right\}$. 
We now present formal results that underlie the fluid approach. The proof of these results essentially follows Kurtz's theory and either explicitly or implicitly relies on the properties of the function $f(\boldsymbol{x}, \boldsymbol{l})$.

Lemma 1. Let $\left\{\boldsymbol{n}^{[v]}(t) \mid v \in \mathbb{N}_{>0}\right\}$ be the sample paths of an infinite sequence of closed QNs whose $v$-th element is the modified model scaled with a total population of $v N$ jobs as well as $v$ times service rates and $v$ self-looping jobs at each DPS station $i$. The sequence $\left\{\boldsymbol{n}^{[v]}(t) \mid v \in \mathbb{N}_{>0}\right\}$ constitutes a density-dependent family of CTMCs.

Proof. See Appendix B

Lemma 2. Define a vector field $\boldsymbol{F}: \mathbb{R}^{R} \rightarrow \mathbb{R}^{R}$ by

$$
\boldsymbol{F}(\boldsymbol{x})=\sum_{\boldsymbol{l}} \boldsymbol{l} f(\boldsymbol{x}, \boldsymbol{l})
$$

Specifically,

$$
F_{i, k, r}(\boldsymbol{x})=\sum_{j=1}^{M} \sum_{l=1}^{K} \sum_{s=1}^{R_{j, l}}\left(f\left(\boldsymbol{x},-\boldsymbol{e}_{j, l, s}+\boldsymbol{e}_{i, k, r}\right)-f\left(\boldsymbol{x},-\boldsymbol{e}_{i, k, r}+\boldsymbol{e}_{j, l, s}\right)\right) .
$$

The initial value problem (IVP)

$$
\left\{\begin{array}{l}
\frac{d \boldsymbol{x}(t)}{d t}=\boldsymbol{F}(\boldsymbol{x}(t)) \\
\boldsymbol{x}(0)=\boldsymbol{n}(0)
\end{array}\right.
$$

has a unique solution $\boldsymbol{x}(t)$ in any finite time horizon $T$.

Proof. See Appendix C.

Theorem 1. Given the same initial state

$$
\frac{\boldsymbol{n}^{[v]}(0)}{v}=\boldsymbol{n}(0)
$$

the normalized sequence $\left\{\boldsymbol{n}^{[v]}(t) / v \mid v \in \mathbb{N}_{>0}\right\}$ converges to the solution $\boldsymbol{x}(t)$ to the IVP (7) in the sense that for any small number $\delta>0$,

$$
\lim _{v \rightarrow \infty} \mathbb{P}\left(\sup _{t \in[0, T]}\left\|\frac{\boldsymbol{n}^{[v]}(t)}{v}-\boldsymbol{x}(t)\right\|>\delta\right)=0 .
$$

Proof. See Appendix D,

Corollary 1. Suppose that the condition of Theorem 1 is satisfied. The expectation of the normalized sequence $\left\{\boldsymbol{n}^{[v]}(t) / v \mid v \in \mathbb{N}_{>0}\right\}$ converges to the solution $\boldsymbol{x}(t)$ to the IVP (7) in any finite time horizon T:

$$
\lim _{v \rightarrow \infty} \mathbb{E}\left(\frac{\boldsymbol{n}^{[v]}(t)}{v}\right)=\boldsymbol{x}(t) \quad \text { for } t \in[0, T]
$$


Corollary 1 implies that as the scaling factor $v$ increments, the expected normalized sample path $\mathbb{E}\left(\boldsymbol{n}^{[v]}(t) / v\right)$ of the $v$-th model gradually approaches the solution $\boldsymbol{x}(t)$ to the fluid ODE system. As stated in Lemma (1), we scale the total population of jobs as well as service rates and self-looping jobs at each DPS station. Since this scaling scheme preserves the actual completion

$$
Q_{i, k}(t)=\sum_{r=1}^{R_{i, k}} \mathbb{E}\left(n_{i, k, r}(t)\right) \approx \sum_{r=1}^{R_{i, k}} x_{i, k, r}(t) \quad \text { for } t \in[0, T] .
$$

Any function $g\left(\boldsymbol{w}_{i}\right)$ in compliance with $(2)$ gives an optional form for the service weight $w_{i, 0}$ of the extra self-looping job at station $i$. We recommend the reader to use the arithmetic mean over the entries of the local service weight vector $\boldsymbol{w}_{i}$ :

$$
w_{i, 0}=g\left(\boldsymbol{w}_{i}\right)=\frac{\sum_{k=1}^{K} w_{i, k}}{K},
$$

so that the extra self-looping job acts as an average job across all the classes at the station. The validation results presented in Section 6 are based on this intuitive choice. Other forms, for example $\min _{k \in[1 . . K]} w_{i, k}$ and $\max _{k \in[1 . . K]} w_{i, k}$, are feasible and may be chosen in particular instances, but we have observed them to yield less accurate results on average across the set of instances used in the validation and therefore are not considered throughout.

(11) shows that the solution $\boldsymbol{x}(t)$ to the fluid ODE system forms an approximation to the expected sample path $\mathbb{E}(\boldsymbol{n}(t))$ of our reference model on any finite time interval $[0, T]$. The transient mean queue length of class- $k$ jobs at station $i$ can thus be written as

To obtain the steady-state value, (11) needs to be extended at infinite time $t \rightarrow \infty$ :

$$
\lim _{t \rightarrow \infty} \mathbb{E}(\boldsymbol{n}(t)) \approx \lim _{t \rightarrow \infty} \boldsymbol{x}(t)=\tilde{\boldsymbol{x}},
$$

where $\tilde{\boldsymbol{x}}$ is the attractor of the fluid ODE system. Applying (15) yields

$$
\tilde{Q}_{i, k}=\sum_{r=1}^{R_{i, k}} \lim _{t \rightarrow \infty} \mathbb{E}\left(n_{i, k, r}(t)\right) \approx \sum_{r=1}^{R_{i, k}} \tilde{x}_{i, k, r} .
$$


The proof of 15) essentially requires showing that the fluid ODE system admits a unique attractor $220 \quad \tilde{x}$ :

$$
\lim _{t \rightarrow \infty} \boldsymbol{x}(t)=\tilde{\boldsymbol{x}}
$$

and that the expected normalized sample path $\boldsymbol{n}^{[v]}(t) / v$ converges to the solution $\boldsymbol{x}(t)$ to the fluid ODE system at infinite time $t \rightarrow \infty$ :

$$
\lim _{t \rightarrow \infty} \lim _{v \rightarrow \infty} \mathbb{E}\left(\frac{\boldsymbol{n}^{[v]}(t)}{v}\right)=\lim _{t \rightarrow \infty} \boldsymbol{x}(t),
$$

which are known to be difficult. In practice, we replace the attractor $\tilde{\boldsymbol{x}}$ with a solution point $\boldsymbol{x}\left(t_{\infty}\right)$ founded by evolving the fluid ODE system until all the derivatives nearly vanish. Although this is not completely rigorous from a theoretical point of view, no failed cases have been encountered in the large set of model instances created for validation.

\section{Advanced Applications}

The fluid approach features the ability to analyze the transient behavior of a given model. This is useful, for example, to capture the dynamics of a random environment between successive stage changes [32]. Transient analysis also enables approximation of response time distributions at a station where the service of an incoming job starts immediately after arrival [20]. In what follows, we introduce a refined method and its extension for approximating response time distributions at individual stations and for the entire system, building on the concept of chains [19].

\subsection{Concept of Chains}

The routing probability matrix $\boldsymbol{P}=\left[P_{(i, k),(j, l)}\right]$ can be considered as defining a discrete-time Markov chain (DTMC) whose states are station-class pairs of form $(i, k)$. Assuming the routing DTMC to be decomposable into ergodic subchains, Muntz [19] showed in the product-form case that a closed QN with multiple chains is equivalent to one with the same number of classes. The states of an ergodic DTMC are reachable pairwise from each other, recurrent non-null and aperiodic. If we represent the routing DTMC as a weighted directed graph, then each chain manifests itself as a strongly connected subgraph. Since all the chains are completely isolated from one another, it is impossible for a job to switch between different chains. Let $\mathcal{V}_{q}$ and $\mathcal{E}_{q}$ be the sets of station-class pairs and connections in chain $q$ respectively. The population of chain- $q$ jobs is thus

$$
N_{q}=\sum_{(i, k) \in \mathcal{V}_{q}} \sum_{r=1}^{R_{i, k}} n_{i, k, r}
$$

For convenience, we denote by $\mathcal{C}_{i, q}$ the set of classes that may be visited by a chain- $q$ job at station $i$, and by $\mathcal{C}_{q}$ the set of all classes that may be visited by a chain- $q$ job, i.e. $\mathcal{C}_{i, q}=\left\{k \mid(i, k) \in \mathcal{V}_{q}\right\}$ and $\mathcal{C}_{q}=\bigcup_{i=1}^{M} \mathcal{C}_{i, q}$. The concept of chains is essential not only in that our reference model is 
defined as a multi-chain QN but also in that it underlies the methods for approximating response time distributions.

\subsection{Response Time Distributions}

To approximate the response time distribution for class $k$ at station $i$, the method present in 20] tags all class- $k$ jobs at that station and restore them upon service completion. Consequently, the duration that a class- $k$ job is being tagged corresponds to its residual sojourn time at station $i$, which differs from its response time there unless the service time of the job is memoryless, i.e. exponentially distributed. Our method overcomes this limitation by adding a start phase in the service time distribution for class $k$ at station $i$ to tag class- $k$ jobs that have just arrived at the station.

First, we evaluate the mean service time of a class- $k$ job at station $i$ by applying the moment formula for phase-type distributions:

$$
S_{i, k}=\boldsymbol{\alpha}_{i, k}\left(-\boldsymbol{T}_{i, k}\right)^{-1} \mathbf{1}_{i, k}
$$

where $\boldsymbol{\alpha}_{i, k}=\left(\alpha_{i, k, r}\right) \in \mathbb{R}^{1 \times R_{i, k}}$ and $\boldsymbol{T}_{i, k}=\left[T_{i, k,(r, s)}\right] \in \mathbb{R}^{R_{i, k} \times R_{i, k}}$ are the initial probability vector and the transition rate matrix that represent the service time distribution for class $k$ at station $i$, and $\mathbf{1}_{i, k} \in \mathbb{N}^{R_{i, k} \times 1}$ is a column vector of all ones. The $(r, s)$-th entry of transition rate matrix $\boldsymbol{T}_{i, k}$ is given by

$$
T_{i, k,(r, s)}= \begin{cases}-\mu_{i, k, r} & \text { if } r=s \\ \mu_{i, k, r} P_{i, k,(r, s)} & \text { otherwise. }\end{cases}
$$

Next, we modify the service time distribution for class $k$ at station $i$ so that any class- $k$ arrival at the station starts in an additional phase 0 with probability $\alpha_{i, k, 0}=1$ and shifts from that phase to phase $r$ with probability $P_{i, k,(0, r)}=\alpha_{i, k, r}$. Phase $(i, k, 0)$ therefore gathers up all class- $k$ jobs having just arrived at station $i$. To limit the side effects of the modification, the service rate of a job in phase $(i, k, 0)$ is set as $\mu_{i, k, 0}=1 / \eta S_{i, k}$, where $\eta$ is a small positive coefficient ${ }^{1}$ After that, we build a fluid ODE system that incorporates the modified service time distribution, and compute its steady-state solution $\tilde{\boldsymbol{x}}^{\prime} \in \mathbb{R}^{R^{\prime}}$ under the initial condition $\boldsymbol{x}^{\prime}(0) \in \mathbb{R}^{R^{\prime}}$, where $R^{\prime}=R+1$. The initial condition $\boldsymbol{x}^{\prime}(0)$ arises from the initial state $\boldsymbol{n}(0)$ by inserting zero entries. Then, we create an auxiliary class $k^{\prime}$ for tagging class- $k$ jobs. Class $k^{\prime}$ has the same service time distribution and the same service weight as class $k$ at station $i$. The probability of a class- $k^{\prime}$ job at station $i$ being forwarded to station $j$ as a class- $l$ job is set to $P_{\left(i, k^{\prime}\right),(j, l)}=P_{(i, k),(j, l)}$. A tagged class- $k$ job is thereby restored immediately after departure from station $i$. Finally, we

\footnotetext{
${ }^{1}$ The positive coefficient $\eta$ cannot be chosen to be arbitrarily small as this will end up with a stiff fluid ODE system and a large computational error.
} 
build another fluid ODE system that incorporates the auxiliary class 2 and compute its transient solution $\boldsymbol{x}^{\prime \prime}(t) \in \mathbb{R}^{R^{\prime \prime}}$ under the initial condition $\boldsymbol{x}^{\prime \prime}(0) \in \mathbb{R}^{R^{\prime \prime}}$, where $R^{\prime \prime}=R+R_{i, k^{\prime}}+2$. The initial condition $\boldsymbol{x}^{\prime \prime}(0)$ derives from the resultant steady-state solution $\tilde{\boldsymbol{x}}^{\prime}$ by inserting zero entries except that $x_{i, k, 0}^{\prime \prime}(0)=0$ and $x_{i, k^{\prime}, 0}^{\prime \prime}(0)=\tilde{x}_{i, k, 0}^{\prime}$.

$$
\sum_{n=1}^{N_{q}} \mathbb{1}_{\left\{\forall u \in[0, t], y_{q, n}(u) \in \mathcal{P}_{i, k} \wedge y_{q, n}(0)=(i, k, 0)\right\}}=\sum_{r=0}^{R_{i, k^{\prime}}} n_{i, k^{\prime}, r}^{\prime \prime}(t) .
$$

Suppose that $(i, k)$ is a station-class pair in chain $q$, i.e. $(i, k) \in \mathcal{V}_{q}$. Because any class- $k$ job at station $i$ belongs to chain $q$ and because all chain- $q$ jobs are indistinguishable from one another, the probability of the response time of a class- $k$ job at station $i$ being longer than time span $t$ is equal to that of the $n$-th job in chain $q$ staying at station $i$ as a class- $k$ job during time interval $[0, t]$ after being observed in phase $(i, k, 0)$ at time 0 :

$$
\mathbb{P}\left(\tilde{r p}_{i, k}>t\right) \approx \mathbb{P}\left(\forall u \in[0, t], y_{q, n}(u) \in \mathcal{P}_{i, k} \mid y_{q, n}(0)=(i, k, 0)\right)
$$

where $y_{q, n}(u)$ is the phase visited by the $n$-th job in chain $q$ at time $u$, and $\mathcal{P}_{i, k}$ is the set of phases in the service time distribution for class $k$ at station $i$, i.e. $\mathcal{P}_{i, k}=\left\{(i, k, r) \mid r \in\left[0 \ldots R_{i, k}\right]\right\}$. Particularly, the approximate equality in $(23)$ is due to the addition of phase $(i, k, 0)$. The count of events that a chain- $q$ job is in phase $(i, k, 0)$ at time 0 and stays as a class- $k$ job at station $i$ during time interval $[0, t]$ is exactly the number of class- $k^{\prime}$ jobs at station $i$ at time $t$. That is,

Taking the expectation on both sides of $(24)$ and dividing the results by $N_{q}$ leads to

$$
\begin{aligned}
& \mathbb{P}\left(\forall u \in[0, t], y_{q, n}(u) \in \mathcal{P}_{i, k} \wedge y_{q, n}(0)=(i, k, 0)\right) \\
= & \mathbb{E}\left(\mathbb{1}_{\left\{\forall u \in[0, t], y_{q, n}(u) \in \mathcal{P}_{i, k} \wedge y_{q, n}(0)=(i, k, 0)\right\}}\right) \\
= & \frac{1}{N_{q}} \mathbb{E}\left(\sum_{n=1}^{N_{q}} \mathbb{1}_{\left\{\forall u \in[0, t], y_{q, n}(u) \in \mathcal{P}_{i, k} \wedge y_{q, n}(0)=(i, k, 0)\right\}}\right) \\
= & \frac{\mathbb{E}\left(\sum_{r=0}^{R_{i, k^{\prime}}} n_{i, k^{\prime}, r}^{\prime \prime}(t)\right)}{N_{q}}=\frac{\sum_{r=0}^{R_{i, k^{\prime}}} \mathbb{E}\left(n_{i, k^{\prime}, r}^{\prime \prime}(t)\right)}{N_{q}} .
\end{aligned}
$$

Substituting $t$ with 0 in 25 and noticing that all class- $k^{\prime}$ jobs at station $i$ are in phase 0 at time

\footnotetext{
${ }^{2}$ Auxiliary classes are essentially a tool for tagging jobs that satisfy certain conditions. The service weights for such classes should not be taken into account in that of a self-looping job at any DPS station.
} 
0 , we get

$$
\begin{aligned}
& \mathbb{P}\left(y_{q, n}(0)=(i, k, 0)\right)=\mathbb{P}\left(y_{q, n}(0) \in \mathcal{P}_{i, k} \wedge y_{q, n}(0)=(i, k, 0)\right) \\
= & \frac{\sum_{r=0}^{R_{i, k^{\prime}}} \mathbb{E}\left(n_{i, k^{\prime}, r}^{\prime \prime}(0)\right)}{N_{q}}=\frac{\mathbb{E}\left(n_{i, k^{\prime}, 0}^{\prime \prime}(0)\right)}{N_{q}} .
\end{aligned}
$$

295

It follows from 22, 23, 25, 26) and (11) that

$$
\begin{aligned}
\Phi_{i, k}(t)= & 1-\mathbb{P}\left(\tilde{r p}_{i, k}>t\right) \\
& \approx 1-\mathbb{P}\left(\forall u \in[0, t], y_{q, n}(u) \in \mathcal{P}_{i, k} \mid y_{q, n}(0)=(i, k, 0)\right) \\
= & 1-\frac{\mathbb{P}\left(\forall u \in[0, t], y_{q, n}(u) \in \mathcal{P}_{i, k} \wedge y_{q, n}(0)=(i, k, 0)\right)}{\mathbb{P}\left(y_{q, n}(0)=(i, k, 0)\right)} \\
= & 1-\frac{\sum_{r=0}^{R_{i, k^{\prime}}} \mathbb{E}\left(n_{i, k^{\prime}, r}^{\prime \prime}(t)\right)}{\mathbb{E}\left(n_{i, k^{\prime}, 0}^{\prime \prime}(0)\right)} \approx 1-\frac{\sum_{r=0}^{R_{i, k^{\prime}}} x_{i, k^{\prime}, r}^{\prime \prime}(t)}{x_{i, k^{\prime}, 0}^{\prime \prime}(0)}
\end{aligned}
$$

27) provides an approximation to the response time CDF for class $k$ at station $i$ using the transient solution $\boldsymbol{x}^{\prime \prime}(t)$.

\subsection{System Response Time Distributions}

System performance measures of a closed QN with class switching are evaluated per chain with 300 respect to a reference point or station [33. We only consider the second case, where the system response time of a job, for example, is defined as the end-to-end time that the job spends traveling through the QN excluding its think time at the reference station. Like the earlier version [20], the refined method is further extensible to approximate the system response time distribution for chain $q$ with respect to a delay station $D$. The basic idea is to add an end phase in the service time distribution for each class $k \in \mathcal{C}_{D, q}$ at station $D$ to tag chain- $q$ jobs that are about to depart from the station and restore them upon next arrival. Thus, our extension is generally applicable in the sense that the total service time of a chain- $q$ job needs not to be nearly exponentially distributed.

First, we evaluate the mean system response time of a chain- $q$ job with respect to station $D$ by applying Little's law to chain $q$ at the system level:

$$
\tilde{R} p_{q-D}=\frac{\tilde{Q}_{q-D}}{\tilde{X}_{q-D}},
$$

310

where $\tilde{Q}_{q-D}$ and $\tilde{X}_{q-D}$ are the system mean queue length and the system average throughput of chain- $q$ jobs with respect to station $D$. The former can be obtained from 16 as

$$
\tilde{Q}_{q-D}=N_{q}-\sum_{k \in \mathcal{C}_{D, q}} \tilde{Q}_{D, k} \approx N_{q}-\sum_{k \in \mathcal{C}_{D, q}} \sum_{r=1}^{R_{D, k}} \tilde{x}_{D, k, r} .
$$

Applying Little's law to each class $k \in \mathcal{C}_{D, q}$ at station $D$ together with 16 and noticing that 
station $D$ is of delay type yields the latter:

$$
\tilde{X}_{q-D}=\sum_{k \in \mathcal{C}_{D, q}} \tilde{X}_{D, k}=\sum_{k \in \mathcal{C}_{D, q}} \frac{\tilde{Q}_{D, k}}{\tilde{R} p_{D, k}} \approx \sum_{k \in \mathcal{C}_{D, q}} \frac{\sum_{r=1}^{R_{D, k}} \tilde{x}_{D, k, r}}{S_{D, k}} .
$$

Next, we modify the service time distribution for each class $k \in \mathcal{C}_{D, q}$ at station $D$ so that any class-

$k$ departure from the station shifts from phase $r$ to an additional phase $R_{D, k}+1$ with probability $P_{D, k,\left(r, R_{D, k}+1\right)}=\beta_{D, k, r}$ and ends in that phase with probability $\beta_{D, k, R_{D, k}+1}=1$. The service rate of a job in phase $\left(D, k, R_{D, k}+1\right)$ is set as $\mu_{D, k, R_{D, k}+1}=1 / \eta \tilde{R} p_{q-D}$. Since $\eta$ is a small positive coefficient 1 , phase $\left(D, k, R_{D, k}+1\right)$ gathers up all class- $q$ jobs about to depart from station $D$ as a class- $k$ job. After that, we build a fluid ODE system that incorporates the modified service time distributions, and compute its steady-state solution $\tilde{\boldsymbol{x}}^{\prime} \in \mathbb{R}^{R^{\prime}}$ under the initial condition $\boldsymbol{x}^{\prime}(0) \in \mathbb{R}^{R^{\prime}}$, where $R^{\prime}=R+\left|\mathcal{C}_{D, q}\right|$. The initial condition $\boldsymbol{x}^{\prime}(0)$ arises from the initial state $\boldsymbol{n}(0)$ by inserting zero entries. Then, we create $\left|\mathcal{C}_{q}\right|$ auxiliary classes for tagging chain- $q$ jobs. Each auxiliary class $k^{\prime}$ corresponds to a class $k \in \mathcal{C}_{q}$ and has the same service time distribution and the same service weight as class $k$ at every station $i$ such that $(i, k)$ is a station-class pair in chain $q$, i.e. $(i, k) \in \mathcal{V}_{q}$. For each connection $((i, k),(j, l)) \in \mathcal{E}_{q}$, the probability of a class- $k^{\prime}$ job at station $i$ being forwarded to station $j=D$ as a class- $l$ job is set as $P_{\left(i, k^{\prime}\right),(D, l)}=P_{(i, k),(D, l)}$, whilst that of it being forwarded to station $j \neq D$ as a class- $l^{\prime}$ job is set as $P_{\left(i, k^{\prime}\right),\left(j, l^{\prime}\right)}=P_{(i, k),(j, l)}$. A tagged chain- $q$ job is thereby not restored until immediately before next arrival at station $D$. Finally, we build another fluid ODE system that incorporates the auxiliary classes ${ }^{2}$, and compute its transient solution $\boldsymbol{x}^{\prime \prime}(t) \in \mathbb{R}^{R^{\prime \prime}}$ under the initial condition $\boldsymbol{x}^{\prime \prime}(0) \in \mathbb{R}^{R^{\prime \prime}}$, where $R^{\prime \prime}=R+\sum_{(i, k) \in \mathcal{V}_{q}} R_{i, k^{\prime}}+2\left|\mathcal{C}_{D, q}\right|$. The initial condition $\boldsymbol{x}^{\prime \prime}(0)$ derives from the resultant steady-state solution $\tilde{\boldsymbol{x}}^{\prime}$ by inserting zero entries except that $x_{D, k, R_{D, k}+1}^{\prime \prime}(0)=0$ and $x_{D, k^{\prime}, R_{D, k^{\prime}}+1}^{\prime \prime}(0)=\tilde{x}_{D, k, R_{D, k}+1}^{\prime}$ for each class $k \in \mathcal{C}_{D, q}$ and its auxiliary class $k^{\prime}$.

Let $\tilde{p}_{q-D}$ be the system response time of chain $q$ with respect to station $D$. Following a derivation similar to that of (27) leads to

$$
\Phi_{q-D}(t)=\mathbb{P}\left(\tilde{r p}_{q-D} \leq t\right) \approx 1-\frac{\sum_{k \in \mathcal{C}_{D, q}} x_{D, k^{\prime}, R_{D, k^{\prime}}+1}^{\prime \prime}(t)+\sum_{\substack{(i, k) \in \mathcal{V}_{q} \\ i \neq D}} \sum_{r=1}^{R_{i, k^{\prime}}} x_{i, k^{\prime}, r}^{\prime \prime}(t)}{\sum_{k \in \mathcal{C}_{D, q}} x_{D, k^{\prime}, R_{D, k^{\prime}}+1}^{\prime \prime}(0)} .
$$

Given the transient solution $\boldsymbol{x}^{\prime \prime}(t)$, we can approximate the system response time CDF for chain $q$ with respect to station $D$ by applying (31).

\section{Validations}

This section reports validations of the fluid approach against simulation for both transient and steady-state analysis in terms of mean queue lengths. In the transient validation, we focus 
on representative examples, given that capturing the transient behavior of a model instance by simulation is time-consuming. As for the steady-state validation, the fluid approach is compared with the standard approximate mean value analysis (AMVA) algorithm for DPS given in [34] on a large set of model instances. We have also validated the refined method for approximating

\subsection{Model Parameter Settings}

The validation experiments are designed on a set of 4914 model instances, which are created by altering the topology $\left(P_{(i, k),(j, l)}\right)$, service time distributions $\left(R_{i, k}, \alpha_{i, k, r}, P_{i, k,(r, s)}, \beta_{i, k, r}\right)$, number of stations $(M)$, number of classes $(K)$, total population of jobs $(N)$, service rates $\left(\mu_{i, k, r}\right)$, service weights $\left(w_{i, k}\right)$ and random seed $\left(\mu_{i, k, r}, w_{i, k}\right)$. The topology of a model instance is either serial or parallel with one delay station, as illustrated in Figures 1 and 2. Because the steady-state mean queue lengths appears insensitive to the types of service time distributions for our reference model, we choose service times to be exponentially distributed for the entire set of model instances but consider additional representative examples with Coxian service time distributions when necessary. Each model instance consists of 3,5 or 9 stations and 2, 4 or 8 classes with a total population of 128,256 or 512 jobs, which are divided equally among all the classes and initialized at the delay station. For simplicity, the probability of a departure from the delay station moving to any DPS station is set the same, and no class switching is allowed between any two classes so that each class constitutes a separate chain. Service rates are either balanced and unbalanced for a model instance. In the balanced case, we let service rates at all the DPS stations be 5.0. As to the unbalanced case, a collection of $K$ values is sampled from a uniform distribution with interval $[1.0,9.0]$, and we use these values as service rates at every DPS station ${ }^{3}$. Service weights are generated in a similar way, except that the unbalanced are totally randomized across different DPS station $\$^{3}$ When service rates or service weights are unbalanced, multiple model instances are obtained by incrementing the random seed from 1 to 30. Particularly, service rates at the delay station are always balanced and determined such that the average utilization of every DPS station would be 0.9375 if there was a total population of 128 jobs with balanced service rates and balanced service weights. This is to ensure that $(12)$ holds for most of the model instances at least in the steady state. Table 1 lists model parameter settings for the validations.

\footnotetext{
${ }^{3}$ Given a model instance with $\mathrm{M}$ stations and $\mathrm{K}$ classes, we generate unbalanced service rates and unbalanced service weights at the DPS stations through the MATLAB commands 8 *gallery ('uniformdata', $[1, \mathrm{~K}]$, seed) +1 and $8 *$ gallery ('uniformdata' $[\mathrm{M}-1, \mathrm{~K}], 10000+$ seed) +1 respectively, where seed is the random seed of that model instance.
} 


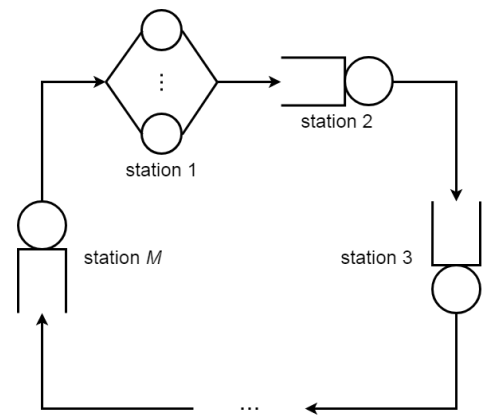

Figure 1: The serial topology.

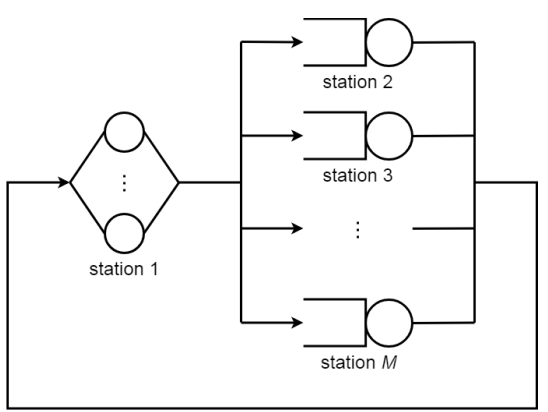

Figure 2: The parallel topology.

Table 1: Model parameter settings for the validations.

\begin{tabular}{lll}
\hline Dimension & Optional Values & Related Parameters \\
\hline Topology & Serial, Parallel & $P_{(i, k),(j, l)}$ \\
Service Time Distributions & Exponential, Coxian* & $R_{i, k}, \alpha_{i, k, r}, P_{i, k,(r, s)}, \beta_{i, k, r}$ \\
Number of Stations & $3 \dagger 5,9^{\dagger}$ & $M$ \\
Number of Classes & $2 \dagger^{\dagger} 4,8^{\dagger}$ & $K$ \\
Total Population of Jobs & $1288^{\dagger} 256,512^{\dagger}$ & $N$ \\
Service Rates & Balanced: $5.0 \dagger$ Unbalanced: $[1.0,9.0]$ & $\mu_{i, k}$ \\
Service Weights & Balanced: $5.0 \dagger$ Unbalanced: $[1.0,9.0]$ & $w_{i, k}$ \\
Random Seed & $1,22^{\dagger} \ldots, 30^{\dagger}$ & $\mu_{i, k}, w_{i, k}$ \\
\hline
\end{tabular}

$* \dagger \ddagger$ These values are exclusive to the validations for transient, steady-state and response time distribution analysis respectively.

\subsection{Tool Parameter Settings}

We use MATLAB (R2017a) as the platform to run the validation experiments. The fluid ODE system for each model instance is solved numerically with the help of ode23, a non-stiff execution of ode23 programmatically by specifying an event that stops the evolution of the fluid ODE system upon occurrence. Two open-source tools, JMT [35] and LINE [20], are also invoked from MATLAB. JMT is an integrated framework for performance evaluation, capacity planning and workload characterization of computer and communication systems based on the queueing theory. A suite of six Java applications are available in JMT, including a discrete-event simulator called JSIM. In a recent release of JMT (v1.0.2), an extension to JSIM have been developed to support simulation of DPS stations. LINE is a MATLAB toolbox for performance and reliability analysis of systems and processes that can be described by queueing models. The AMVA algorithm for DPS given by Lazowska et al. 34] has been recently implemented in the corresponding solver 
of LINE (v2.0.0). Additionally, LINE provides a programming interface to build and run model instances in JSIM. Table 2 lists tool parameter settings for the validations.

Table 2: Tool parameter settings for the validations.

\begin{tabular}{lll}
\hline Solver & Critical Parameter & Specified Value \\
\hline \multirow{2}{*}{ MATLAB - ode23 } & Relative Tolerance & $10^{-9}$ \\
& Absolute Tolerance & $10^{-9}$ \\
& Time Span & {$\left[0,10^{5}\right]$} \\
& Stop Event I & $\|d \boldsymbol{x}(t) / d t\|_{\infty} \leq 10^{-3}$ for 100 times $^{* \dagger}$ \\
& Stop Event II & $1-\Phi_{i, k}(t) \leq 10^{-3 \ddagger} 1-\Phi_{q-D}(t) \leq 10^{-3 \ddagger}$ \\
\hline \multirow{3}{*}{ JMT - JSIM } & Random Seed & Random \\
& Maximum Simulation Time & $t_{\infty}^{*}{ }^{*}$ Infinity ${ }^{\dagger} 800 t_{\infty}^{\ddagger}$ \\
& Automatic Stop & Disabled \\
& Maximum Samples & $1.6 \times 10^{8} / K$ \\
\hline \multirow{2}{*}{ LINE - AMVA } & Iteration Tolerance & $10^{-6}$ \\
& Maximum Iterations & $10^{3}$ \\
\hline
\end{tabular}

$* \dagger \ddagger$ These values are exclusive to the validations for transient, steady-state and response time distribution analysis respectively.

\subsection{Transient Analysis}

The accuracy of the fluid approach for transient analysis is evaluated using the following error metric:

$$
\max _{i \in[1 . . M], k \in[1 . . K]} \frac{\left|Q_{i, k}^{\mathrm{app}}(t)-Q_{i, k}^{\mathrm{sim}}(t)\right|}{N_{k}} \text { for } t \in[0, T],
$$

390

where $Q_{i, k}^{\mathrm{app}}(t)$ is the approximated transient mean queue length and $Q_{i, k}^{\mathrm{sim}}(t)$ is the corresponding simulated result. This metric tells the maximum percentage of misplaced jobs resulting from fluid approximation relative to simulation at time $t \in[0, T]$.

The validation experiments for transient analysis are performed on four representative examples. We select a serial and a parallel example from the considered set of model instances, both having exponential service time distributions, 5 stations, 4 classes, a total population of 256 jobs, unbalanced service rates, unbalanced service weights and random seed 1. The other two examples are created by changing service time distributions from exponential to 2-phase Coxian with coefficient of variation 2 .

To capture the trajectory of the fluid ODE system for each example, we stop the execution of ode23 when the maximum norm of the derivative $d \boldsymbol{x}(t) / d t$ is no more than $10^{-3}$ for 100 times. The transient mean queue lengths are approximated by applying (14) with the resultant trajectory. 
The end time of this trajectory, denoted by $t_{\infty}$, is further used as the maximum simulation time of JSIM to control the length of simulation. The simulated results are acquired by averaging the sample paths of queue lengths over 2000 repeated simulations.

Figure 3 shows the error in using the fluid approach to approximate transient mean queue lengths for each example, which increases over time and converges around a limiting value below 0.1. The approximated and simulated transient mean queue lengths at the first DPS station for each example are shown in Figure 4 Comparing Figures $4 \mathrm{a}$ and $4 \mathrm{c}$ with $4 \mathrm{~b}$ and $4 \mathrm{~d}$ respectively, we observe for our reference model that the types of service time distributions, more technically the higher-order moments, may impact on transient measures but are seemingly a minor factor when it comes to steady-state analysis.

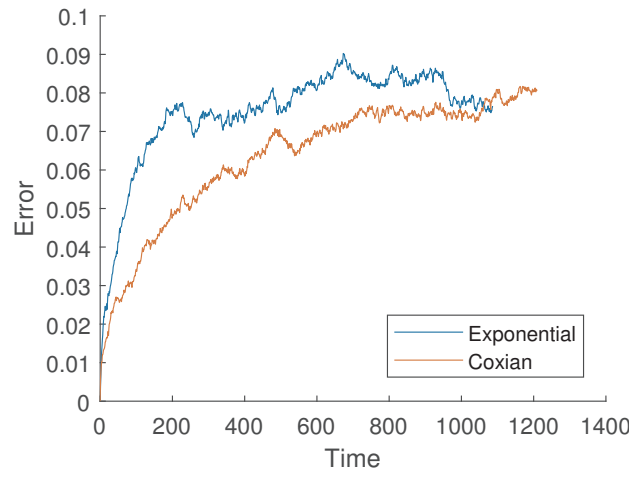

(a) Serial

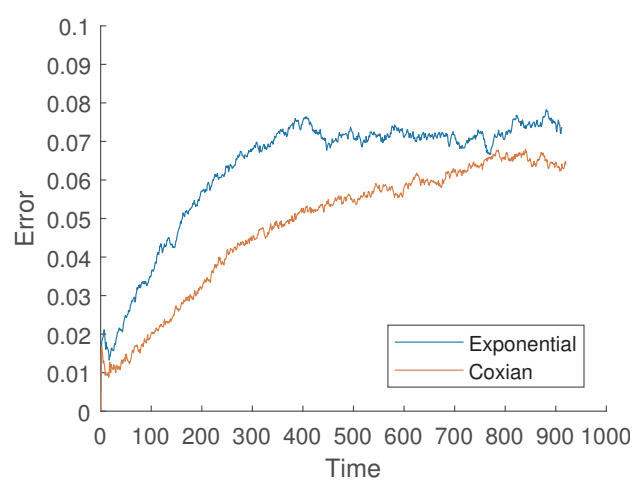

(b) Parallel

Figure 3: The error in using the fluid approach to approximate transient mean queue lengths for each example.

\subsection{Steady-State Analysis}

The accuracy of the fluid approach for steady-state analysis is evaluated in terms of an error metric defined in [36]:

$$
\max _{i \in[1 . . M], k \in[1 . . K]} \frac{\left|\tilde{Q}_{i, k}^{\mathrm{app}}-\tilde{Q}_{i, k}^{\mathrm{sim}}\right|}{N_{k}},
$$

which is natural extension of (32) at infinite time $t \rightarrow \infty$. For comparison, we also evaluate the accuracy of the standard AMVA algorithm for DPS [34. This algorithm determines the average residence time of a class- $k$ job at a DPS queueing station $i$ of a closed QN as

$$
\bar{R}_{i, k}(\boldsymbol{N}) \approx D_{i, k}\left(1+\frac{\sum_{l=1}^{K} w_{i, l} \bar{Q}_{i, l}\left(\boldsymbol{N}-\boldsymbol{e}_{k}\right)}{w_{i, k}}\right),
$$

where $D_{i, k}$ is the service demand of a class- $k$ job at the station, $\bar{Q}_{i, l}\left(\boldsymbol{N}-\boldsymbol{e}_{k}\right)$ is the mean queue length of class-l jobs at the station when the model has one less class- $k$ job. 

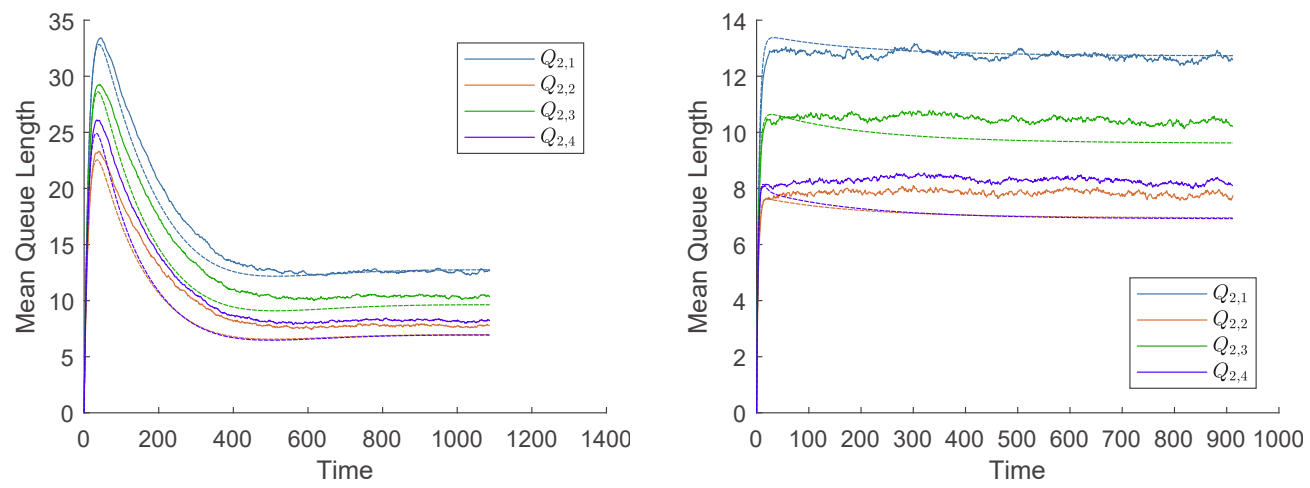

(a) Serial - Exponential

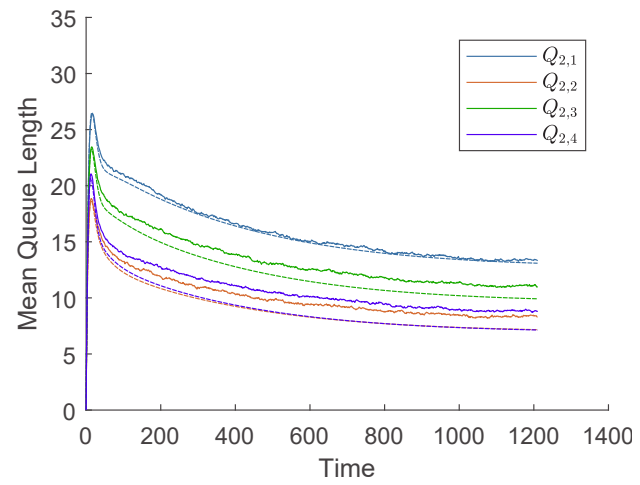

(c) Parallel - Exponential

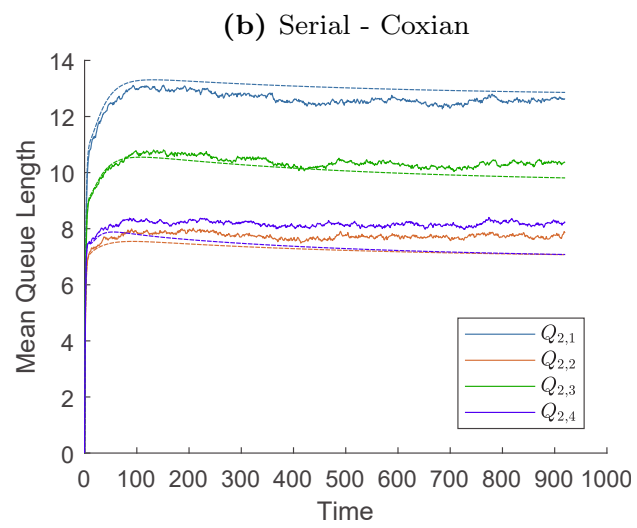

(d) Parallel - Coxian

Figure 4: The approximated and simulated transient mean queue lengths at the first DPS station for each example. Dashed lines indicate fluid approximations.

The validation experiments for steady-state analysis are carried out on the entire set of model instances. We use the same stop event as for transient analysis to control the execution of ode23 and obtain the attractor of the fluid ODE system as the end point of the resultant trajectory. The approximated steady-state mean queue length is computed by applying (15). The simulated results are provided by JSIM directly in a long simulation where at least $1.6 \times 10^{8} / \mathrm{K}$ samples are collected for a single measure. The mean queue lengths approximated by the AMVA algorithm are acquired using the corresponding solver in LINE.

Figure 5 shows the average errors in using the average errors in using the fluid approach and the AMVA algorithm to approximate steady-state mean queue lengths for the considered set of model instances. The two techniques perform similarly along different dimensions of the model parameter space. Their overall average errors for a model instance are 0.0557 and 0.0554 , which are almost identical. As shown in Figure 5c, the fluid approach is initially worse but gets increasingly better than the AMVA algorithm as the total population of jobs grows. This reflects a desirable feature of fluid approximation. We further notice from Figure $5 \mathrm{~d}$ that the average errors of both techniques are below 0.01 when service weights are balanced, which means that they are highly 


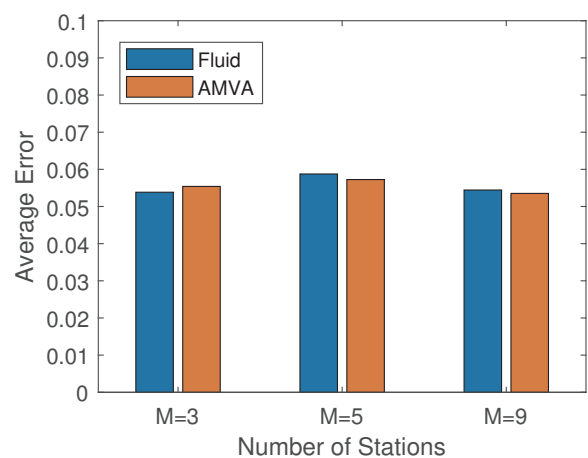

(a)

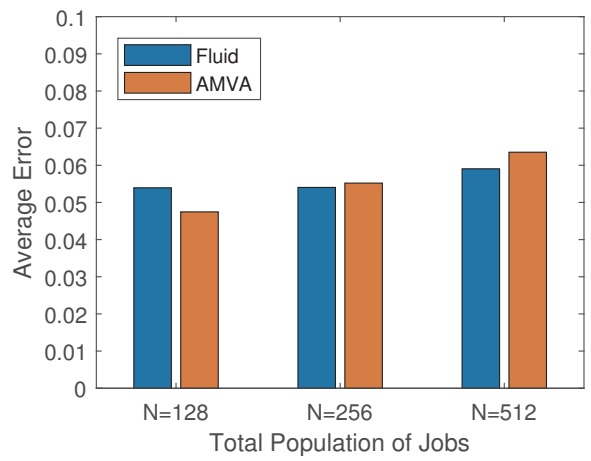

(c)

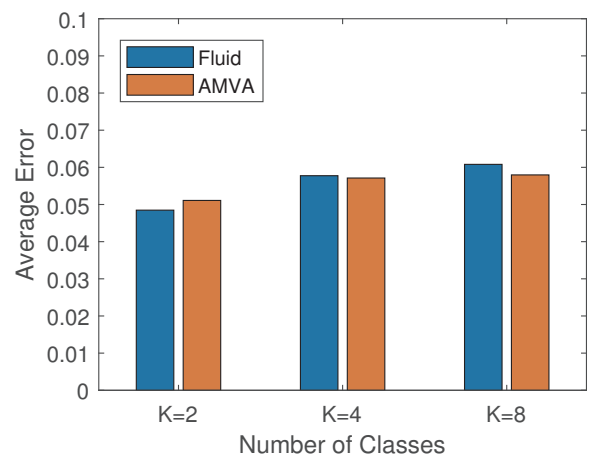

(b)

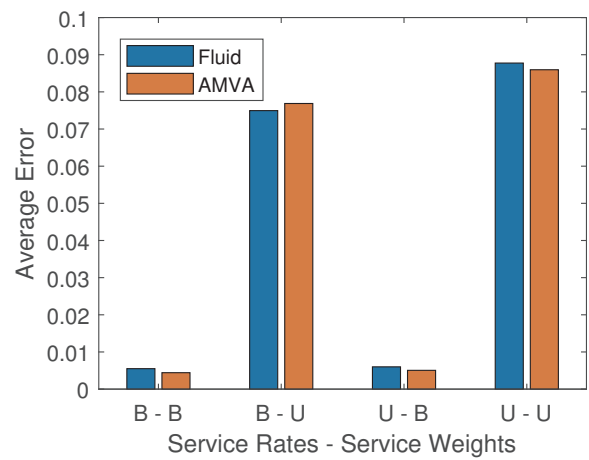

(d)

Figure 5: The average errors in using the fluid approach and the AMVA algorithm to approximate steady-state mean queue lengths for the considered set of model instances.

$$
\max _{i \in[1 . . M], k \in[1 . . K]}\left|\left(\Phi_{i, k}^{\mathrm{app}}\right)\left(\left(\Phi_{i, k}^{\mathrm{sim}}\right)^{-1}(0.95)\right)-0.95\right|,
$$

This is the maximum difference between the compared and reference response time CDFs at the 
95-th percentile across all the stations and classes. Similarly, the error metric for evaluating the resulting system response time CDFs for a model instance is defined as follows:

$$
\max _{q \in[1 . . U]}\left|\left(\Phi_{q-D}^{\mathrm{app}}\right)\left(\left(\Phi_{q-D}^{\mathrm{sim}}\right)^{-1}(0.95)\right)-0.95\right|,
$$

which is the maximum difference between the compared and reference system response time CDFs at the 95-th percentile across all the chains.

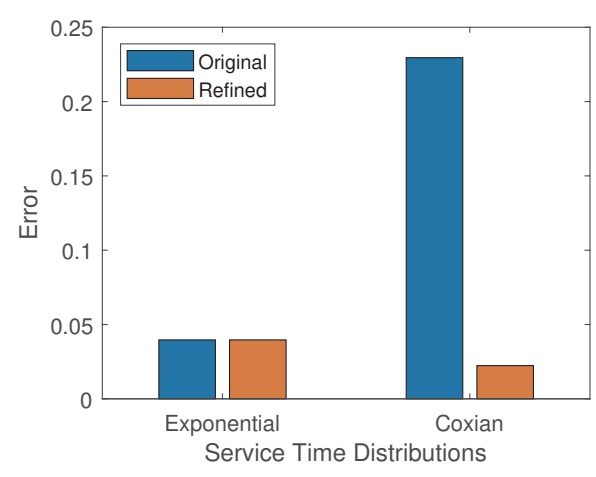

(a) Serial

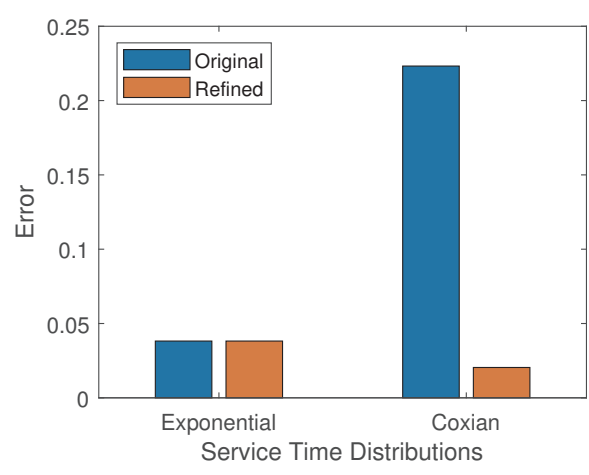

(b) Parallel

Figure 6: The errors in using the original and the refined method to approximate station response time distributions for each example.

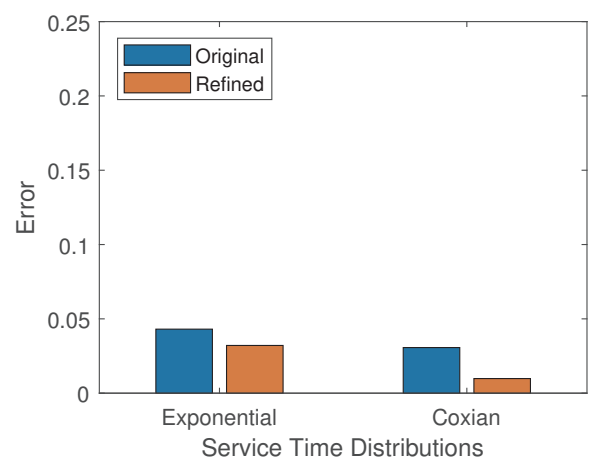

(a) Serial

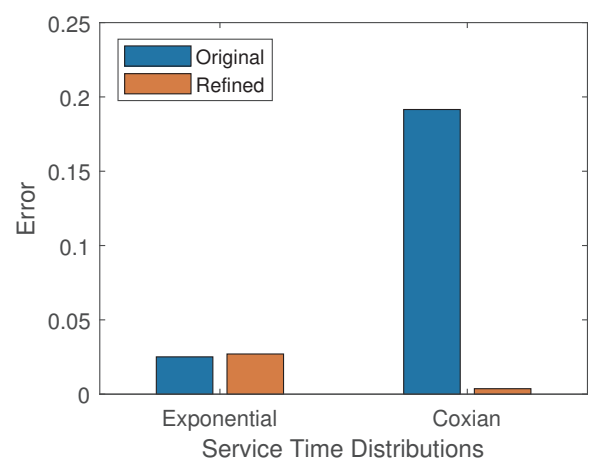

(b) Parallel

Figure 7: The errors in using the original and the refined method to approximate system response time distributions for each example.

Figure 6 and Figure 7 illustrates the results of the application validation. The error of the refined method in approximating either station or system response time distributions is below 0.5 for each typical example, which is generally less than that of the original method. When the service time distributions are exponential, the two methods exhibits the same accuracy at the station level. This is because the refined method reduces to the original one in this case. By contrast, it becomes much more accurate for the example with Coxian service time distributions, 


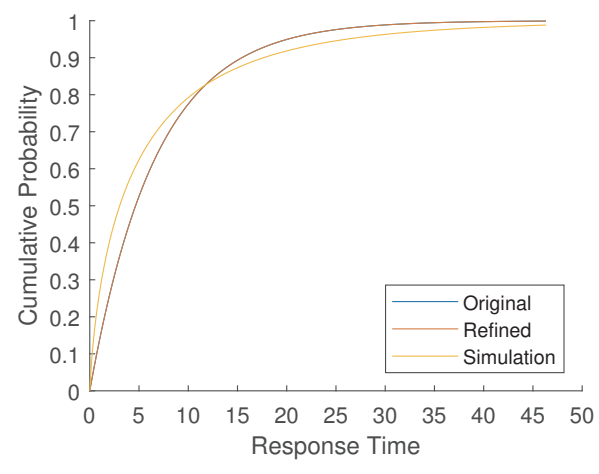

(a) Exponential

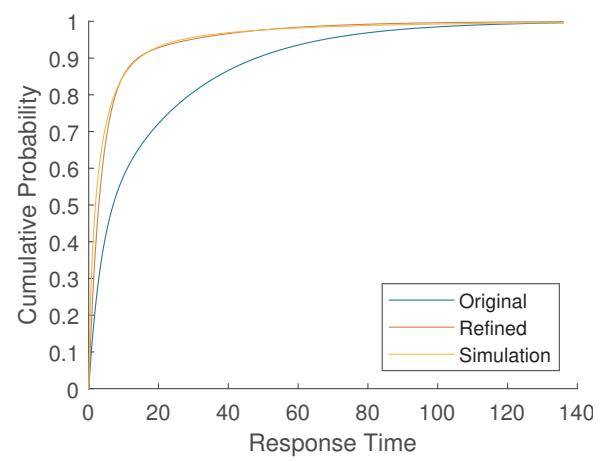

(b) Coxian

Figure 8: The approximated and simulated response time CDFs of class-1 jobs at the first DPS station for serial examples.

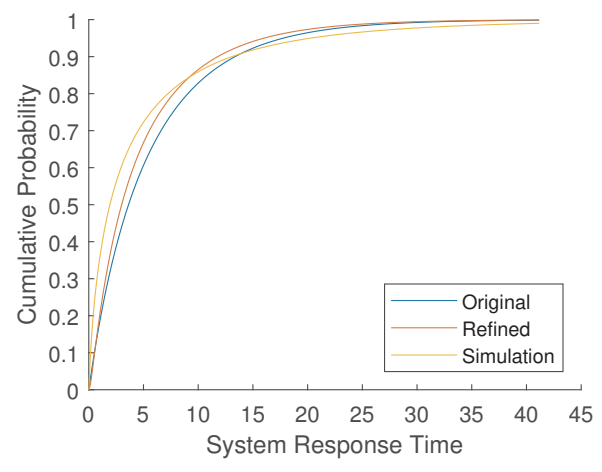

(a) Exponential

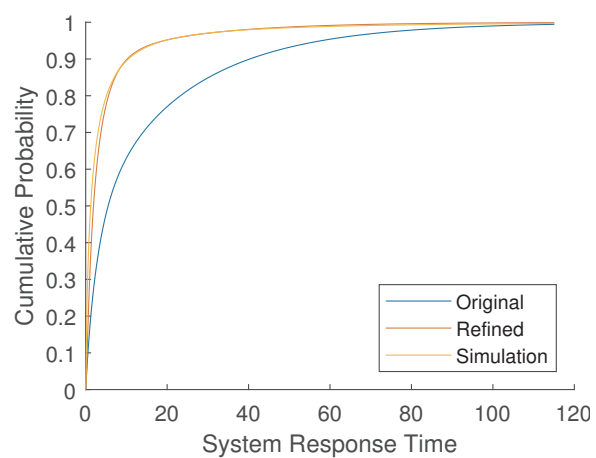

(b) Coxian

Figure 9: The approximated and simulated response time CDFs of chain-1 jobs with respect to the delay station for parallel examples.

where the assumption of the original method fails. Both methods provide good approximations at the system level for typical examples with a serial topology. The original method is slightly better than the refined one for the example with a parallel topology and exponential service time distributions. Further investigation finds that all the system response time distributions in this example happens to have a coefficient of variation that is nearly 1 and can be regarded as being exponential. For instance, Figure 8 illustrates the resulting response time CDFs for class 1 at station 2 (DPS) in typical examples with a serial topology, while those for chain 1 with respect to station 1 (delay) are shown in Figure 9 It can be seen that the refined method always exhibits a good agreement with simulation both at individual stations and for the entire system but large discrepancies may exist between the original method and simulation. 


\subsection{Summary}

Based on the above findings, we believe that the fluid approach can deliver good results for (RADON).

\section{Appendices}

\section{A. Main Notation}


Table A.1: General notation.

\begin{tabular}{cl}
\hline Symbol & Definition \\
\hline$\wedge$ & Logical conjunction \\
$\vee$ & Logical disjunction \\
$\forall$ & Universal quantification \\
$\exists$ & Existential quantification \\
\hline $\mathbb{R}^{*}$ & Set of real numbers \\
$\mathbb{Z}^{*}$ & Set of whole numbers \\
$\mathbb{N}^{*}$ & Set of natural numbers \\
\hline$(a, b)$ & Open real interval between $a$ and $b$ \\
{$[a, b]$} & Closed real interval between $a$ and $b$ \\
$(a . . b)$ & Open integer interval between $a$ and $b$ \\
{$[a \ldots b]$} & Closed integer interval between $a$ and $b$ \\
\hline $\mathbb{P}(A)$ & Probability of event $A$ \\
$\mathbb{E}(X)$ & Expectation of random variable $X$ \\
$\mathbb{1} A$ & Indicator of event $A$ \\
$\|\boldsymbol{x}\|^{\dagger}$ & Norm of vector $\boldsymbol{x}$ \\
\hline
\end{tabular}

* $\mathbb{R}_{P}^{n}$ represents the $n$-dimensional vector space of real numbers that satisfy predicate $P$, e.g. $\mathbb{R}_{>0}^{N}=$ $\prod_{i=1}^{N}\{x \in R \mid x>0\}$. Similar notation also applies to the integer sets $\mathbb{Z}$ and $\mathbb{N}$.

$\dagger\|\boldsymbol{x}\|$ alone denotes an arbitrary norm of vector $\boldsymbol{x}$, which must be positive definite, subadditive and absolutely homogeneous. If subscripted with a real number $p \geq 1$, it specifically refers to the $p$-norm of vector $\boldsymbol{x}$, i.e. $\|\boldsymbol{x}\|_{p}=\left(\sum_{i}\left|x_{i}\right|^{p}\right)^{1 / p}$.

Table A.2: Contextual notation.

\begin{tabular}{cl}
\hline Symbol & Definition \\
\hline$i, j$ & Station indices \\
$k, l$ & Class indices \\
$r, s$ & Phase indices \\
$q$ & Chain index \\
\hline$M$ & Number of stations \\
$K$ & Number of classes \\
$U$ & Number of chains \\
$N$ & Total population of jobs
\end{tabular}




\begin{tabular}{|c|c|}
\hline $\mathcal{D}$ & Set of delay stations \\
\hline $\mathcal{Q}$ & Set of DPS stations \\
\hline$R_{i, k}{ }^{*}$ & $\begin{array}{l}\text { Number of phases composing the service time distribution of a class- } k \text { job at } \\
\text { station } i\end{array}$ \\
\hline$\mu_{i, k, r}$ & Service rate of a class- $k$ job in phase $r$ at station $i$ \\
\hline$\alpha_{i, k, r}$ & Probability that a class- $k$ job at station $i$ starts in phase $r$ \\
\hline$P_{i, k,(r, s)}$ & Probability that a class- $k$ job at station $i$ shifts from phase $r$ to phase $s$ \\
\hline$\beta_{i, k, r}$ & Probability that a class- $k$ job at station $i$ ends in phase $r$ \\
\hline$w_{i, k}{ }^{*}$ & Service weight of a class- $k$ job at station $i$ \\
\hline$P_{(i, k),(j, l)}$ & $\begin{array}{l}\text { Probability that a class- } k \text { job at station } i \text { switches to class } l \text { and moves to } \\
\text { station } j\end{array}$ \\
\hline$P_{(i, k, r),(j, l, s)}$ & $\begin{array}{l}\text { Probability that a class- } k \text { job in phase } r \text { at station } i \text { proceeds to station } j \text { as a } \\
\text { class- } l \text { job in phase } s\end{array}$ \\
\hline$n_{i, k, r}$ & Number of class- $k$ jobs in phase $r$ at station $i$ \\
\hline$x_{i, k, r}$ & Amount of class- $k$ fluid in phase $r$ at station $i$ \\
\hline$N_{k}^{\dagger}$ & Population of class- $k$ jobs \\
\hline$S_{i, k}$ & Service requirement of a class- $k$ job at station $i$ \\
\hline$D_{i, k}$ & Service demand of a class- $k$ job at station $i$ \\
\hline$\tilde{Q}_{i, k}$ & Mean queue length of class- $k$ jobs at station $i$ \\
\hline$\tilde{R} p_{i, k}$ & Average response time of a class- $k$ job at station $i$ \\
\hline$\tilde{R} d_{i, k}$ & Average residence time of a class- $k$ job at station $i$ \\
\hline$N_{q}$ & Population of chain- $q$ jobs \\
\hline$\tilde{Q}_{q-i}$ & Mean system queue length of chain- $q$ jobs with respect to station $i$ \\
\hline$\tilde{R} p_{q-i}$ & Average system response time of a chain- $q$ job with respect to station $i$ \\
\hline$\tilde{X}_{q-i}$ & Average system throughput of chain- $q$ jobs with respect to station $i$ \\
\hline \multicolumn{2}{|c|}{$\begin{array}{l}{ }^{*} R_{i, k} \text { and } w_{i, k} \text { may be ambiguous for a station-class pair }(i, k) \text {. In these cases, our convention is to let } R_{i, k}=0 \\
\text { and } w_{i, k}=0 \text { respectively. }\end{array}$} \\
\hline $\begin{array}{l}\dagger \\
N_{k} \text { becomes } \\
\text { such that } k=\end{array}$ & $\begin{array}{l}\text { deterministic only when there is no class switching, i.e. } P_{(i, k),(j, l)}=0 \text { for all classes } k, l \in[1 \ldots K] \\
\qquad l \text {. }\end{array}$ \\
\hline
\end{tabular}

\section{B. Proof of Lemma 1}

The elements of the sequence $\left\{\boldsymbol{n}^{[v]}(t) \mid v \in \mathbb{N}_{>0}\right\}$ represent closed QNs with a multiple scale of the modified model. According to the scaling scheme, the set of possible state changes in each of these models remains invariant, i.e. $L^{[v]}=L=\left\{-\boldsymbol{e}_{i, k, r}+\boldsymbol{e}_{j, l, s} \mid i, j \in[1 \ldots M], k, l \in[1 \ldots K], r \in\right.$ $\left.\left[1 \ldots R_{i, k}\right], s \in\left[1 \ldots R_{j, l}\right]\right\}$, and the transition rate of the $v$-th model from state $\boldsymbol{n}$ to state $\boldsymbol{n}+\boldsymbol{l}$ is 
given by

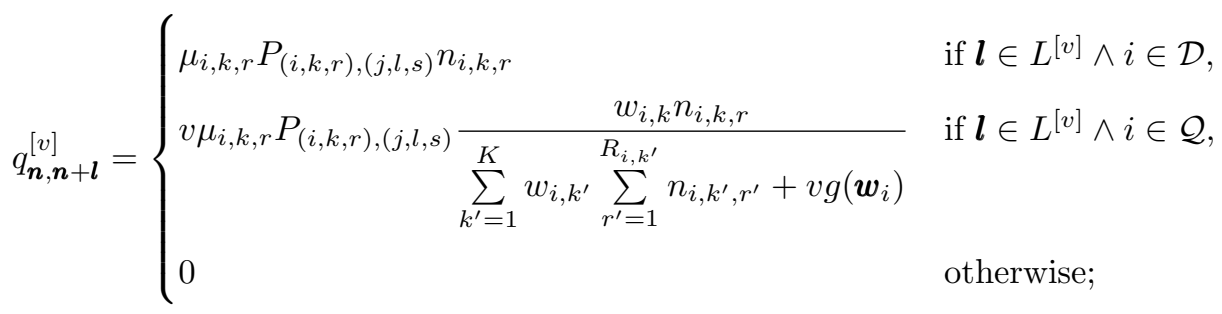

$$
\begin{aligned}
& = \begin{cases}v \mu_{i, k, r} P_{(i, k, r),(j, l, s)} \frac{n_{i, k, r}}{v} & \text { if } \boldsymbol{l} \in L \wedge i \in \mathcal{D}, \\
v \mu_{i, k, r} P_{(i, k, r),(j, l, s)} \frac{w_{i, k} \frac{n_{i, k, r}}{v}}{\sum_{k^{\prime}=1}^{K} w_{i, k^{\prime}} \sum_{r^{\prime}=1}^{R_{i, k^{\prime}}} \frac{n_{i, k^{\prime}, r^{\prime}}}{v}+g\left(\boldsymbol{w}_{i}\right)} & \text { if } \boldsymbol{l} \in L \wedge i \in \mathcal{Q}, \\
0 & \text { otherwise. }\end{cases}
\end{aligned}
$$

Comparing (B.1) with (4) yields

$$
q_{\boldsymbol{n}, \boldsymbol{n}+\boldsymbol{l}}^{[v]}=v f\left(\frac{\boldsymbol{n}}{v}, \boldsymbol{l}\right) .
$$

(B.2) implies that for any state change $\boldsymbol{l}$, the transition rate of the $v$-th model in state $\boldsymbol{n}$ can be expressed as $v$ times that of the first model in state $\boldsymbol{n} / v$. The sequence $\left\{\boldsymbol{n}^{[v]}(t) \mid v \in \mathbb{N}_{>0}\right\}$ therefore constitutes a density-dependent family of CTMCs [16].

\section{Proof of Lemma 2}

The modified model is a closed QN with a total population of $N$ jobs across all the phases. The entries of its sample path $\boldsymbol{n}(t)$ must be non-negative and sum up to $N$ :

$$
\left\{\begin{array}{l}
n_{i, k, r}(t) \geq 0, \\
\sum_{i=1}^{M} \sum_{k=1}^{K} \sum_{r=1}^{R_{i, k}} n_{i, k, r}(t)=N .
\end{array}\right.
$$

This is also true for each element of the normalized sequence $\left\{\boldsymbol{n}^{[v]}(t) / v \mid v \in \mathbb{N}_{>0}\right\}$ and every candidate solution $\boldsymbol{x}(t)$ to the IVP (7):

$$
\left\{\begin{array}{l}
\frac{n_{i, k, r}^{[v]}(t)}{v} \geq 0 \\
\sum_{i=1}^{M} \sum_{k=1}^{K} \sum_{r=1}^{R_{i, k}} \frac{n_{i, k, r}^{[v]}(t)}{v}=N
\end{array}\right.
$$

515

$$
\left\{\begin{array}{l}
x_{i, k, r}(t) \geq 0, \\
\sum_{i=1}^{M} \sum_{k=1}^{K} \sum_{r=1}^{R_{i, k}} x_{i, k, r}(t)=N .
\end{array}\right.
$$

The former piece of C.3 holds because

$$
x_{i, k, r}(0)=n_{i, k, r}(0) \geq 0
$$


and

$$
\begin{aligned}
& \left.\frac{d x_{i, k, r}(t)}{d t}\right|_{x_{i, k, r}(t)=0}=\left.F_{i, k, r}(\boldsymbol{x}(t))\right|_{x_{i, k, r}(t)=0} \\
= & \left.\sum_{j=1}^{M} \sum_{l=1}^{K} \sum_{s=1}^{R_{j, l}}\left(f\left(\boldsymbol{x}(t),-\boldsymbol{e}_{j, l, s}+\boldsymbol{e}_{i, k, r}\right)-f\left(\boldsymbol{x}(t),-\boldsymbol{e}_{i, k, r}+\boldsymbol{e}_{j, l, s}\right)\right)\right|_{x_{i, k, r}(t)=0} \\
= & \left.\sum_{j=1}^{M} \sum_{l=1}^{K} \sum_{s=1}^{R_{j, l}} f\left(\boldsymbol{x}(t),-\boldsymbol{e}_{j, l, s}+\boldsymbol{e}_{i, k, r}\right)\right|_{x_{i, k, r}(t)=0} \geq 0 .
\end{aligned}
$$

(C.4) and (C.5) result from (7), (C.1), (6) and (4). The latter piece of (C.3) is due to the fact that

$$
\sum_{i=1}^{M} \sum_{k=1}^{K} \sum_{r=1}^{R_{i, k}} x_{i, k, r}(0)=\sum_{i=1}^{M} \sum_{k=1}^{K} \sum_{r=1}^{R_{i, k}} n_{i, k, r}(0)=N
$$

520

and that

$$
\begin{aligned}
& \frac{d \sum_{i=1}^{M} \sum_{k=1}^{K} \sum_{r=1}^{R_{i, k}} x_{i, k, r}(t)}{d t}=\sum_{i=1}^{M} \sum_{k=1}^{K} \sum_{r=1}^{R_{i, k}} \frac{d x_{i, k, r}(t)}{d t}=\sum_{i=1}^{M} \sum_{k=1}^{K} \sum_{r=1}^{R_{i, k}} F_{i, k, r}(\boldsymbol{x}(t)) \\
= & \sum_{i, j=1}^{M} \sum_{k, l=1}^{K} \sum_{r=1}^{R_{i, k}} \sum_{s=1}^{R_{j, l}}\left(f\left(\boldsymbol{x}(t),-\boldsymbol{e}_{j, l, s}+\boldsymbol{e}_{i, k, r}\right)-f\left(\boldsymbol{x}(t),-\boldsymbol{e}_{i, k, r}+\boldsymbol{e}_{j, l, s}\right)\right)=0 .
\end{aligned}
$$

(C.6) and (C.7) derive from (7), (C.1) and (6).

Consider an open set $E=\left\{\boldsymbol{x} \in \mathbb{R}_{>-\epsilon}^{R}|| \sum_{i=1}^{M} \sum_{k=1}^{K} \sum_{r=1}^{R_{i, k}} x_{i, k, r}-N \mid<1\right\}$, where $0<\epsilon<$ $\min _{i \in \mathcal{Q}} g\left(\boldsymbol{w}_{i}\right) / \sum_{k^{\prime}=1}^{K} w_{i, k^{\prime}} R_{i, k^{\prime}}$. We know from C.3 that

$$
\boldsymbol{x}(t) \in E \quad \text { for } t \in[0, T]
$$

The closure of the set $E$ is a non-empty compact and convex subset of the set $D$, which is the global domain of the function $f(\boldsymbol{x}, \boldsymbol{l})$. As in (6), each entry of the vector field $\boldsymbol{F}(\boldsymbol{x})$ is a linear combination of the function $f(\boldsymbol{x}, \boldsymbol{l})$ at relevant state changes $\boldsymbol{l} \in L$. The vector field $\boldsymbol{F}(\boldsymbol{x})$ thus retains the continuity, boundedness and Lipschitz continuity of the function $f(\boldsymbol{x}, \boldsymbol{l})$ on the set $E$ :

$$
\begin{gathered}
\lim _{\boldsymbol{x}^{\prime} \rightarrow \boldsymbol{x}} \boldsymbol{F}\left(\boldsymbol{x}^{\prime}\right)=\boldsymbol{F}(\boldsymbol{x}) \quad \text { for } \boldsymbol{x} \in E ; \\
\|\boldsymbol{F}(\boldsymbol{x})\| \leq M_{E} \quad \text { for } \boldsymbol{x} \in E ; \\
\left\|\boldsymbol{F}\left(\boldsymbol{x}^{\prime}\right)-\boldsymbol{F}(\boldsymbol{x})\right\| \leq K_{E}\left\|\boldsymbol{x}^{\prime}-\boldsymbol{x}\right\| \quad \text { for } \boldsymbol{x}^{\prime}, \boldsymbol{x} \in E .
\end{gathered}
$$

530 $c_{1}, c_{2}>0$ such that for any vector $\boldsymbol{a} \in \mathbb{R}^{R}$,

$$
c_{1}\|\boldsymbol{a}\|_{1} \leq\|\boldsymbol{a}\| \leq c_{2}\|\boldsymbol{a}\|_{1}
$$


Let $\boldsymbol{x}_{0}$ be an arbitrary state satisfying (C.3). From (C.12, (C.9), C.10) and (C.11), we can find a closed neighborhood of the state $\boldsymbol{x}_{0}, B_{0}=\left\{\boldsymbol{x} \in \mathbb{R}^{R} \mid\left\|\boldsymbol{x}-\boldsymbol{x}_{0}\right\| \leq c_{1} \epsilon / 2\right\} \subset\left\{\boldsymbol{x} \in \mathbb{R}^{R} \mid\left\|\boldsymbol{x}-\boldsymbol{x}_{0}\right\|_{1} \leq\right.$ $\epsilon / 2\} \subset E$, on which the vector field $\boldsymbol{F}(\boldsymbol{x})$ is continuous and has a bound $M_{B_{0}} \leq M_{E}$ as well as ${ }_{535}$ a Lipschitz constant $K_{B_{0}} \leq K_{E}$. It then follows by the Picard-Lindelöf theorem (Theorem 8.13 in [37]) that the ODE system in (7) is uniquely solvable under the initial condition $\boldsymbol{x}\left(t_{0}\right)=\boldsymbol{x}_{0}$ on the time interval $\left[t_{0}, t_{0}+\Delta\right]$, where $\Delta=c_{1} \epsilon / 2 M_{E} \leq c_{1} \epsilon / 2 M_{B_{0}}$. This result is applicable to a finite sequence of intersecting time intervals $\left\{[\nu \Delta,(\nu+1) \Delta] \mid \nu \in \mathbb{N}_{<T / \Delta\}}\right.$. The IVP (7) therefore has a unique solution $\boldsymbol{x}(t)$ in any finite time horizon $T$.

\section{Proof of Theorem 1}

Applying the triangle inequality and $\mathrm{C} .12$ gives

$$
\left\|-\boldsymbol{e}_{i, k, r}+\boldsymbol{e}_{j, l, s}\right\| \leq\left\|-\boldsymbol{e}_{i, k, r}\right\|+\left\|\boldsymbol{e}_{j, l, s}\right\| \leq c_{2}\left\|-\boldsymbol{e}_{i, k, r}\right\|_{1}+c_{2}\left\|\boldsymbol{e}_{j, l, s}\right\|_{1}=2 c_{2} .
$$

From (4), we can show that

$$
\begin{aligned}
\sup _{\boldsymbol{x} \in E} \sum_{\boldsymbol{l}}\|\boldsymbol{l}\| f(\boldsymbol{x}, \boldsymbol{l}) & =\sup _{\boldsymbol{x} \in E} \sum_{i, j=1}^{M} \sum_{k, l=1}^{K} \sum_{r=1}^{R_{i, k}} \sum_{s=1}^{R_{j, l}}\left\|-\boldsymbol{e}_{i, k, r}+\boldsymbol{e}_{j, l, s}\right\| f\left(\boldsymbol{x},-\boldsymbol{e}_{i, k, r}+\boldsymbol{e}_{j, l, s}\right) \\
& \leq \sup _{\boldsymbol{x} \in E} \sum_{i, j=1}^{M} \sum_{k, l=1}^{K} \sum_{r=1}^{R_{i, k}} \sum_{s=1}^{R_{j, l}} 2 c_{2} f\left(\boldsymbol{x},-\boldsymbol{e}_{i, k, r}+\boldsymbol{e}_{j, l, s}\right)<\infty .
\end{aligned}
$$

where the first inequality results from (D.1), and the second one is due to the boundedness of the function $f(\boldsymbol{x}, \boldsymbol{l})$ on set $E$. Applying (4) and (D.1), we also have

$$
\lim _{d \rightarrow \infty} \sup _{\boldsymbol{x} \in E} \sum_{\|\boldsymbol{l}\|>d}\|\boldsymbol{l}\| f(\boldsymbol{x}, \boldsymbol{l})=\lim _{d \rightarrow \infty} \sup _{\boldsymbol{x} \in E} \sum_{\left\|-\boldsymbol{e}_{i, k, r}+\boldsymbol{e}_{j, l, s}\right\|>d}\left\|-\boldsymbol{e}_{i, k, r}+\boldsymbol{e}_{j, l, s}\right\| f\left(\boldsymbol{x},-\boldsymbol{e}_{i, k, r}+\boldsymbol{e}_{j, l, s}\right)=0 .
$$

By Kurtz's theorem (Theorem 3.1 in [16]) together with Lemmas 1 and 2, it follows from (C.11), (D.2), D.3, C.8 and (8) that the normalized sequence $\left\{\boldsymbol{n}^{[v]}(t) / v \mid v \in \mathbb{N}_{>0}\right\}$ converges to the solution $\boldsymbol{x}(t)$ to the IVP (7) in the sense of (9).

\section{E. Proof of Corollary 1}

Observe that

$$
0 \leq \mathbb{P}\left(\left\|\frac{\boldsymbol{n}^{[v]}(t)}{v}-\boldsymbol{x}(t)\right\|>\delta\right) \leq \mathbb{P}\left(\sup _{t^{\prime} \in[0, T]}\left\|\frac{\boldsymbol{n}^{[v]}\left(t^{\prime}\right)}{v}-\boldsymbol{x}\left(t^{\prime}\right)\right\|>\delta\right) \quad \text { for } t \in[0, T] .
$$

By the squeeze theorem, we obtain from (E.1) and (9)

$$
\lim _{v \rightarrow \infty} \mathbb{P}\left(\left\|\frac{\boldsymbol{n}^{[v]}(t)}{v}-\boldsymbol{x}(t)\right\|>\delta\right)=0 \quad \text { for } t \in[0, T]
$$


Applying the triangle inequality, C.12, C.2 and (C.3) leads to

$$
\begin{aligned}
\left\|\frac{\boldsymbol{n}^{[v]}(t)}{v}-\boldsymbol{x}(t)\right\| & \leq\left\|\frac{\boldsymbol{n}^{[v]}(t)}{v}\right\|+\|\boldsymbol{x}(t)\| \leq c_{2}\left\|\frac{\boldsymbol{n}^{[v]}(t)}{v}\right\|_{1}+c_{2}\|\boldsymbol{x}(t)\|_{1} \\
& =c_{2} \sum_{i=1}^{M} \sum_{k=1}^{K} \sum_{r=1}^{R_{i, k}} \frac{n_{i, k, r}^{[v]}(t)}{v}+c_{2} \sum_{i=1}^{M} \sum_{k=1}^{K} \sum_{r=1}^{R_{i, k}} x_{i, k, r}(t)=2 c_{2} N .
\end{aligned}
$$

By a corollary to the dominated convergence theorem (Corollary 1 to Theorem 1.C in [38]), it follows from E.2 and E.3 that

$$
\lim _{v \rightarrow \infty} \mathbb{E}\left(\frac{\boldsymbol{n}^{[v]}(t)}{v}\right)=\mathbb{E}(\boldsymbol{x}(t))=\boldsymbol{x}(t) \quad \text { for } t \in[0, T]
$$

The expectation of the normalized sequence $\left\{\boldsymbol{n}^{[v]}(t) / v \mid v \in \mathbb{N}_{>0}\right\}$ therefore converges to the solution $\boldsymbol{x}(t)$ to the IVP $(7)$ in any finite time horizon $T$.

\section{F. Simple Example}

Consider the closed QN illustrated in Figure F.1. where a delay station interacts with a DPS station. There are two classes in the QN, each with an initial population of 5 jobs. The think time $e^{\mathrm{F.1}}$ distributions for these classes are exponential with rates 0.2 and 0.5 respectively. The

service time of a class-1 job is sampled from a hyperexponential distribution with initial probability vector $(0.5,0.5)$ and phase rate vector $(0.2,1)$, while that of a class-2 job follows a Coxian distribution with exit probability vector $(0.7,1)$ and phase rate vector $(0.5,0.1)$. The ratio between service weights assigned to a class-1 and a class-2 jobs is $1: 2$. Each departure from the delay station remains in the same class with probability 0.6 or otherwise switches to the other one with probability 0.4. However, no class switching is allowed for departures from the DPS station.

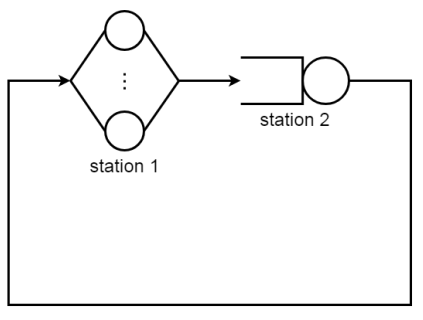

Figure F.1: A simple closed QN.

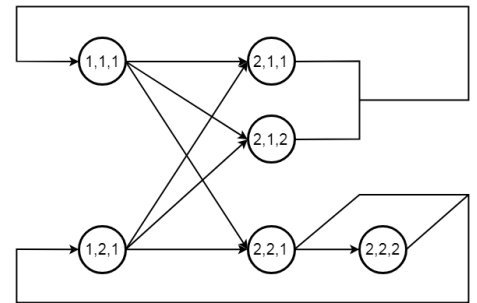

Figure F.2: Job flows between the phases.

To apply the fluid approach, we first formalize the above description by the notation summarized in Appendix A:

- $M=2, K=2, N=10$

\footnotetext{
F.1 Think time here refers to the time that a job spends per visit to the delay station. Note that our techniques do not distinguish between think and service times.
} 
- $\mathcal{D}=\{1\}, \mathcal{Q}=\{2\}$

- $R_{1,1}=1, R_{1,2}=1, R_{2,1}=2, R_{2,2}=2$;

- $\mu_{1,1,1}=0.2, \mu_{1,2,1}=0.5, \mu_{2,1,1}=0.2, \mu_{2,1,2}=1.0, \mu_{2,2,1}=0.5, \mu_{2,2,2}=0.1$;

- $\alpha_{1,1,1}=1.0, \alpha_{1,2,1}=1.0, \alpha_{2,1,1}=0.5, \alpha_{2,1,2}=0.5, \alpha_{2,2,1}=1.0, \alpha_{2,2,2}=0.0$

- $P_{1,1,(1,1)}=0.0, P_{1,2,(1,1)}=0.0$, $P_{2,1,(1,1)}=0.0, P_{2,1,(1,2)}=0.0, P_{2,1,(2,1)}=0.0, P_{2,1,(2,2)}=0.0$, $P_{2,2,(1,1)}=0.0, P_{2,2,(1,2)}=0.3, P_{2,2,(2,1)}=0.0, P_{2,2,(2,2)}=0.0$;

- $\beta_{1,1,1}=1.0, \beta_{1,2,1}=1.0, \beta_{2,1,1}=1.0, \beta_{2,1,2}=1.0, \beta_{2,2,1}=0.7, \beta_{2,2,2}=1.0$

- $w_{1,1}=0.0^{\mathrm{F} .2}, w_{1,2}=0.0^{\mathrm{F} .2}, w_{2,1}=1.0, w_{2,2}=2.0$;

- $P_{(1,1),(1,1)}=0.0, P_{(1,1),(1,2)}=0.0, P_{(1,1),(2,1)}=0.6, P_{(1,1),(2,2)}=0.4$, $P_{(1,2),(1,1)}=0.0, P_{(1,2),(1,2)}=0.0, P_{(1,2),(2,1)}=0.4, P_{(1,2),(2,2)}=0.6$, $P_{(2,1),(1,1)}=1.0, P_{(2,1),(1,2)}=0.0, P_{(2,1),(2,1)}=0.0, P_{(2,1),(2,2)}=0.0$, $P_{(2,2),(1,1)}=0.0, P_{(2,2),(1,2)}=1.0, P_{(2,2),(2,1)}=0.0, P_{(2,2),(2,2)}=0.0$

- $n_{1,1,1}(0)=5, n_{1,2,1}(0)=5, n_{2,1,1}(0)=0, n_{2,1,2}(0)=0, n_{2,2,1}(0)=0, n_{2,2,2}(0)=0$.

This model contains a total of six phases: $(1,1,1),(1,2,1),(2,1,1),(2,1,2),(2,2,1)$ and $(2,2,2)$. As shown in Figure F.2, the set of existent state changes in the model is

$$
\left\{\begin{array}{ccccc}
-\boldsymbol{e}_{1,1,1}+\boldsymbol{e}_{2,1,1}, & -\boldsymbol{e}_{1,1,1}+\boldsymbol{e}_{2,1,2}, & -\boldsymbol{e}_{1,1,1}+\boldsymbol{e}_{2,2,1}, & -\boldsymbol{e}_{1,2,1}+\boldsymbol{e}_{2,1,1}, & -\boldsymbol{e}_{1,2,1}+\boldsymbol{e}_{2,1,2}, \\
-\boldsymbol{e}_{1,2,1}+\boldsymbol{e}_{2,2,1}, & -\boldsymbol{e}_{2,1,1}+\boldsymbol{e}_{1,1,1}, & -\boldsymbol{e}_{2,1,2}+\boldsymbol{e}_{1,1,1}, & -\boldsymbol{e}_{2,2,1}+\boldsymbol{e}_{1,2,1}, & -\boldsymbol{e}_{2,2,1}+\boldsymbol{e}_{2,2,2} \\
-\boldsymbol{e}_{2,2,2}+\boldsymbol{e}_{1,2,1} & & &
\end{array}\right\}
$$

The transition probability for each existent state change can be computed from (1) as

$$
\begin{aligned}
& P_{(1,1,1),(2,1,1)}=0.3, P_{(1,1,1),(2,1,2)}=0.3, P_{(1,1,1),(2,2,1)}=0.4, P_{(1,2,1),(2,1,1)}=0.2, P_{(1,2,1),(2,1,2)}=0.2, \\
& P_{(1,2,1),(2,2,1)}=0.6, P_{(2,1,1),(1,1,1)}=1.0, P_{(2,1,2),(1,1,1)}=1.0, P_{(2,2,1),(1,2,1)}=0.7, P_{(2,2,1),(2,2,2)}=0.3, \\
& P_{(2,2,2),(1,2,1)}=1.0 .
\end{aligned}
$$

\footnotetext{
${ }^{\mathrm{F}}{ }^{2}$ See the footnotes of Appendix $\mathrm{A}$
} 
Further applying (4) yields the corresponding transition rate function:

$$
\begin{aligned}
f\left(\boldsymbol{x},-\boldsymbol{e}_{1,1,1}+\boldsymbol{e}_{2,1,1}\right) & =0.06 x_{1,1,1}, f\left(\boldsymbol{x},-\boldsymbol{e}_{1,1,1}+\boldsymbol{e}_{2,1,2}\right)=0.06 x_{1,1,1}, \\
f\left(\boldsymbol{x},-\boldsymbol{e}_{1,1,1}+\boldsymbol{e}_{2,2,1}\right) & =0.08 x_{1,1,1}, f\left(\boldsymbol{x},-\boldsymbol{e}_{1,2,1}+\boldsymbol{e}_{2,1,1}\right)=0.10 x_{1,2,1}, \\
f\left(\boldsymbol{x},-\boldsymbol{e}_{1,2,1}+\boldsymbol{e}_{2,1,2}\right) & =0.10 x_{1,2,1}, f\left(\boldsymbol{x},-\boldsymbol{e}_{1,2,1}+\boldsymbol{e}_{2,2,1}\right)=0.30 x_{1,2,1}, \\
f\left(\boldsymbol{x},-\boldsymbol{e}_{2,1,1}+\boldsymbol{e}_{1,1,1}\right) & =\frac{0.2 x_{2,1,1}}{1.0 \sum_{r=1}^{2} x_{2,1, r}(t)+2.0 \sum_{r=1}^{2} x_{2,2, r}(t)+1.5}, \\
f\left(\boldsymbol{x},-\boldsymbol{e}_{2,1,2}+\boldsymbol{e}_{1,1,1}\right) & =\frac{1.0 x_{2,1,2}}{1.0 \sum_{r=1}^{2} x_{2,1, r}(t)+2.0 \sum_{r=1}^{2} x_{2,2, r}(t)+1.5}, \\
f\left(\boldsymbol{x},-\boldsymbol{e}_{2,2,1}+\boldsymbol{e}_{1,2,1}\right) & =\frac{0.7 x_{2,2,1}}{1.0 \sum_{r=1}^{2} x_{2,1, r}(t)+2.0 \sum_{r=1}^{2} x_{2,2, r}(t)+1.5} \\
f\left(\boldsymbol{x},-\boldsymbol{e}_{2,2,1}+\boldsymbol{e}_{2,2,2}\right) & =\frac{0.3 x_{2,2,1}}{1.0 \sum_{r=1}^{2} x_{2,1, r}(t)+2.0 \sum_{r=1}^{2} x_{2,2, r}(t)+1.5} \\
f\left(\boldsymbol{x},-\boldsymbol{e}_{2,2,2}+\boldsymbol{e}_{1,2,1}\right) & =\frac{0.2 x_{2,2,2}}{1.0 \sum_{r=1}^{2} x_{2,1, r}(t)+2.0 \sum_{r=1}^{2} x_{2,2, r}(t)+1.5} .
\end{aligned}
$$

It follows from (7) and (6) that the fluid ODE system for the model is

$$
\begin{aligned}
& \frac{d x_{1,1,1}(t)}{d t}=\frac{0.2 x_{2,1,1}(t)+1.0 x_{2,1,2}(t)}{1.0 \sum_{r=1}^{2} x_{2,1, r}(t)+2.0 \sum_{r=1}^{2} x_{2,2, r}(t)+1.5}-0.2 x_{1,1,1}(t), \\
& \frac{d x_{1,2,1}(t)}{d t}=\frac{0.7 x_{2,2,1}(t)+0.2 x_{2,2,2}(t)}{1.0 \sum_{r=1}^{2} x_{2,1, r}(t)+2.0 \sum_{r=1}^{2} x_{2,2, r}(t)+1.5}-0.5 x_{1,2,1}(t), \\
& \frac{d x_{2,1,1}(t)}{d t}=0.06 x_{1,1,1}(t)+0.10 x_{1,2,1}(t)-\frac{1.0 \sum_{r=1}^{2} x_{2,1, r}(t)+2.0 \sum_{r=1}^{2} x_{2,2, r}(t)+1.5}{1.0 \sum_{r=1}^{2} x_{2,1, r}(t)+2.0 \sum_{r=1}^{2} x_{2,2, r}(t)+1.5}, \\
& \frac{d x_{2,1,2}(t)}{d t}=0.06 x_{1,1,1}(t)+0.10 x_{1,2,1}(t)-\frac{1.0 x_{2,2,1}(t)}{1.0 \sum_{r=1}^{2} x_{2,1, r}(t)+2.0 \sum_{r=1}^{2} x_{2,2, r}(t)+1.5}, \\
& \frac{d x_{2,2,1}(t)}{d t}=0.08 x_{1,1,1}(t)+0.30 x_{1,2,1}(t)-\frac{0.2}{r(t)}, \\
& \frac{d x_{2,2,2}(t)}{d t}=\frac{0.3 x_{2,2,1}(t)-0.2 x_{2,2,2}(t)}{1.0 \sum_{r=1}^{2} x_{2,1, r}(t)+2.0 \sum_{r=1}^{2} x_{2,2, r}(t)+1.5},
\end{aligned}
$$

whose initial condition is given by

$$
x_{1,1,1}(0)=5, x_{1,2,1}(0)=5, x_{2,1,1}(0)=0, x_{2,1,2}(0)=0, x_{2,2,1}(0)=0, x_{2,2,2}(0)=0 .
$$

As in (11), the trajectory of this ODE system approximates the expected sample path of the model in any finite time horizon. The transient solution to the model can thus be obtained by evolving 
the ODE system from time 0 until the derivatives become negligible. Using the same tool settings as for the validation (see Section 6.2, we plot the transient solution to the model as shown in Figure F.3, where the steady-state solution is readily available at the truncation point:

$$
\tilde{x}_{1,1,1} \approx 0.56, \tilde{x}_{1,2,1} \approx 0.23, \tilde{x}_{2,1,1} \approx 4.17, \tilde{x}_{2,1,2} \approx 0.84, \tilde{x}_{2,2,1} \approx 1.68, \tilde{x}_{2,2,2} \approx 2.51 .
$$

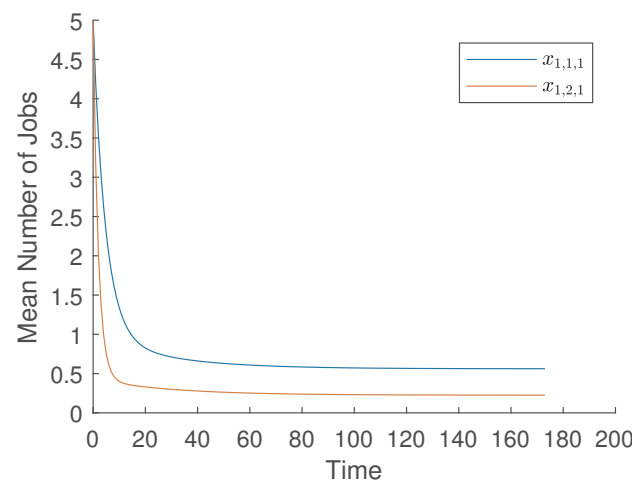

(a) Delay Station

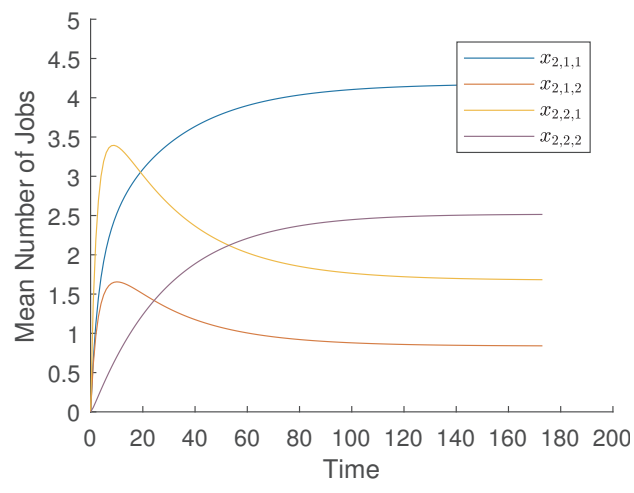

(b) DPS Station

Figure F.3: The transient solution to the model.

From an implementation perspective, it is in fact unnecessary to find the set of existent state

595 Transition probabilities for state changes that actually never occur in the model are zero, resulting in the corresponding transition rate functions being constantly zero-valued. At last, nothing will be contributed to the fluid ODE system by the non-existent state changes.

Figure F.4 shows the routing DTMC of the model, which has in total four states: $(1,1),(1,2)$, class- 1 job at the DPS station is $S_{1,2}=3$. The service rate of a job in the additional phase can thus be set as $\mu_{2,1,0} \approx 11.1$ with the small positive coefficient $\eta=0.03$. Applying the proposed 


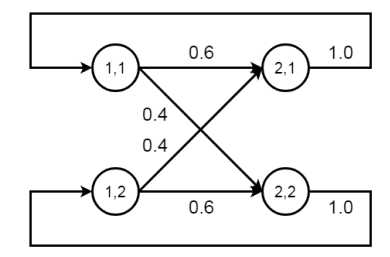

Figure F.4: The routing DTMC of the model.

approach, we obtain the fluid ODE system that incorporates the modified service time distribution:

$$
\begin{aligned}
& \frac{d x_{1,1,1}^{\prime}(t)}{d t}=\frac{0.2 x_{2,1,1}^{\prime}(t)+1.0 x_{2,1,2}^{\prime}(t)}{1.0 \sum_{r=0}^{2} x_{2,1, r}^{\prime}(t)+2.0 \sum_{r=1}^{2} x_{2,2, r}^{\prime}(t)+1.5}-0.2 x_{1,1,1}^{\prime}(t), \\
& \frac{d x_{1,2,1}^{\prime}(t)}{d t}=\frac{0.7 x_{2,2,1}^{\prime}(t)+0.2 x_{2,2,2}^{\prime}(t)}{1.0 \sum_{r=0}^{2} x_{2,1, r}^{\prime}(t)+2.0 \sum_{r=1}^{2} x_{2,2, r}^{\prime}(t)+1.5}-0.5 x_{1,2,1}^{\prime}(t), \\
& \frac{d x_{2,1,0}^{\prime}(t)}{d t}=0.12 x_{1,1,1}^{\prime}(t)+0.20 x_{1,2,1}^{\prime}(t)-\frac{1.0 \sum_{r=0}^{2} x_{2,1, r}^{\prime}(t)+2.0 \sum_{r=1}^{2} x_{2,2, r}^{\prime}(t)+1.5}{d t}, \\
& \frac{d x_{2,1,1}^{\prime}(t)}{d t}=\frac{5.55 x_{2,1,0}^{\prime}(t)-0.2 x_{2,1,1}^{\prime}(t)}{1.0 \sum_{r=0}^{2} x_{2,1, r}^{\prime}(t)+2.0 \sum_{r=1}^{2} x_{2,2, r}^{\prime}(t)+1.5}, \\
& \frac{5.55 x_{2,1,0}^{\prime}(t)-1.0 x_{2,1,2}^{\prime}(t)}{d t}=\frac{1.0 \sum_{r=0}^{2} x_{2,1, r}^{\prime}(t)+2.0 \sum_{r=1}^{2} x_{2,2, r}^{\prime}(t)+1.5}{d x_{2,2,1}^{\prime}(t)}=0.08 x_{1,1,1}^{\prime}(t)+0.30 x_{1,2,1}^{\prime}(t)-\frac{1.0 \sum_{r=0}^{2} x_{2,1, r}^{\prime}(t)+2.0 \sum_{r=1}^{2} x_{2,2, r}^{\prime}(t)+1.5}{d t}, \\
& \frac{d x_{2,2,2}^{\prime}(t)}{d t}=\frac{0.3 x_{2,2,1}^{\prime}(t)-0.2 x_{2,2,2}^{\prime}(t)}{1.0 \sum_{r=0}^{2} x_{2,1, r}^{\prime}(t)+2.0 \sum_{r=1}^{2} x_{2,2, r}^{\prime}(t)+1.5} .
\end{aligned}
$$

The initial condition of the above ODE system is simply

$$
x_{1,1,1}^{\prime}(0)=5, x_{1,2,1}^{\prime}(0)=5, x_{2,1,0}^{\prime}(0)=0, x_{2,1,1}^{\prime}(0)=0, x_{2,1,2}^{\prime}(0)=0, x_{2,2,1}^{\prime}(0)=0, x_{2,2,2}^{\prime}(0)=0 .
$$

Evolving the ODE system until the derivatives almost vanishes yields the steady-state solution $\tilde{\boldsymbol{x}}^{\prime}$ :

$$
\tilde{x}_{1,1,1}^{\prime} \approx 0.56, \tilde{x}_{1,2,1}^{\prime} \approx 0.22, \tilde{x}_{2,1,0}^{\prime} \approx 0.15, \tilde{x}_{2,1,1}^{\prime} \approx 4.11, \tilde{x}_{2,1,2}^{\prime} \approx 0.83, \tilde{x}_{2,2,1}^{\prime} \approx 1.66, \tilde{x}_{2,2,2}^{\prime} \approx 2.48
$$

We then create an auxiliary class $1^{\prime}$ with the same service time distribution and the same service weight as class 1 at the DPS station for tagging class-1 jobs. Figure F.6 shows the routing DTMC 
of the model thereafter. The fluid ODE system that incorporates the auxiliary class is given by

$$
\begin{aligned}
\frac{d x_{1,1,1}^{\prime \prime}(t)}{d t}= & \frac{0.2\left(x_{2,1,1}^{\prime \prime}(t)+x_{2,1^{\prime}, 1}^{\prime \prime}(t)\right)+1.0\left(x_{2,1,2}^{\prime \prime}(t)+x_{2,1^{\prime}, 2}^{\prime \prime}(t)\right)}{1.0 \sum_{r=0}^{2}\left(x_{2,1, r}^{\prime \prime}(t)+x_{2,1^{\prime}, r}^{\prime \prime}(t)\right)+2.0 \sum_{r=1}^{2} x_{2,2, r}^{\prime \prime}(t)+1.5}-0.2 x_{1,1,1}^{\prime \prime}(t), \\
\frac{d x_{1,2,1}^{\prime \prime}(t)}{d t}= & \frac{0.7 x_{2,2,1}^{\prime \prime}(t)+0.2 x_{2,2,2}^{\prime \prime}(t)}{1.0 \sum_{r=0}^{2}\left(x_{2,1, r}^{\prime \prime}(t)+x_{2,1^{\prime}, r}^{\prime \prime}(t)\right)+2.0 \sum_{r=1}^{2} x_{2,2, r}^{\prime \prime}(t)+1.5}-0.5 x_{1,2,1}^{\prime \prime}(t), \\
\frac{d x_{2,1,0}^{\prime \prime}(t)}{d t}= & 0.12 x_{1,1,1}^{\prime \prime}(t)+0.20 x_{1,2,1}^{\prime \prime}(t)-\frac{11.1 x_{2,1,0}^{\prime \prime}(t)}{1.0 \sum_{r=0}^{2}\left(x_{2,1, r}^{\prime \prime}(t)+x_{2,1^{\prime}, r}^{\prime \prime}(t)\right)+2.0 \sum_{r=1}^{2} x_{2,2, r}^{\prime \prime}(t)+1.5}, \\
\frac{d x_{2,1,1}^{\prime \prime}(t)}{d t}= & \frac{5.55 x_{2,1,0}^{\prime \prime}(t)-0.2 x_{2,1,1}^{\prime \prime}(t)}{1.0 \sum_{r=0}^{2}\left(x_{2,1, r}^{\prime \prime}(t)+x_{2,1^{\prime}, r}^{\prime \prime}(t)\right)+2.0 \sum_{r=1}^{2} x_{2,2, r}^{\prime \prime}(t)+1.5}, \\
\frac{d x_{2,1,2}^{\prime \prime}(t)}{d t}= & \frac{5.55 x_{2,1,0}^{\prime \prime}(t)-1.0 x_{2,1,2}^{\prime \prime}(t)}{1.0 \sum_{r=0}^{2}\left(x_{2,1, r}^{\prime \prime}(t)+x_{2,1^{\prime}, r}^{\prime \prime}(t)\right)+2.0 \sum_{r=1}^{2} x_{2,2, r}^{\prime \prime}(t)+1.5}, \\
\frac{d x_{2,2,1}^{\prime \prime}(t)}{d t}= & 0.08 x_{1,1,1}^{\prime \prime}(t)+0.30 x_{1,2,1}^{\prime \prime}(t)-\frac{1.0 \sum_{r=0}^{2}\left(x_{2,1, r}^{\prime \prime}(t)+x_{2,1^{\prime}, r}^{\prime \prime}(t)\right)+2.0 \sum_{r=1}^{\prime \prime} x_{2,2, r}^{\prime \prime}(t)+1.5}{0.3 x_{2,2,1}^{\prime \prime}(t)-0.2 x_{2,2,2}^{\prime \prime}(t)}, \\
\frac{d x_{2,1^{\prime}, 2}^{\prime \prime}(t)}{d t}= & \frac{-11.1 x_{2,1^{\prime}, 0}^{\prime \prime}(t)}{d t}=\frac{1.0 \sum_{r=0}^{2}\left(x_{2,1, r}^{\prime \prime}(t)+x_{2,1^{\prime}, r}^{\prime \prime}(t)\right)+2.0 \sum_{r=1}^{2} x_{2,2, r}^{\prime \prime}(t)+1.5}{\left.1.0 \sum_{r=0}^{2}\left(x_{2,1, r}^{\prime \prime}(t)+x_{2,1^{\prime}, r}^{\prime \prime}(t)\right)+2.0 \sum_{2,1, r}^{\prime \prime} x_{2, r}^{\prime \prime}(t)+x_{2,1^{\prime}, r}^{\prime \prime}(t)\right)+2.0 \sum_{r=1}^{2} x_{2,2, r}^{\prime \prime}(t)+1.5} \\
\frac{d x_{2,1^{\prime}, 0}^{\prime \prime}(t)}{d t}= & \frac{1.0 \sum_{r=0}^{2}\left(x_{2,1, r}^{\prime \prime}(t)+x_{2,1^{\prime}, r}^{\prime \prime}(t)\right)+2.0 \sum_{r=1}^{\prime \prime} x_{2,2, r}^{\prime \prime}(t)+1.5}{5.55 x_{2,1^{\prime}, 0}^{\prime \prime}(t)-0.2 x_{2,1^{\prime}, 1}^{\prime \prime}(t)},
\end{aligned}
$$

${ }_{620}$ Evolving this ODE system under the initial condition

$$
\begin{aligned}
& x_{1,1,1}^{\prime \prime}(0)=0.56, x_{1,2,1}^{\prime \prime}(0)=0.22, x_{2,1,0}^{\prime \prime}(0)=0.00, x_{2,1,1}^{\prime \prime}(0)=4.11, x_{2,1,2}^{\prime \prime}(0)=0.83, \\
& x_{2,2,1}^{\prime \prime}(0)=1.66, x_{2,2,2}^{\prime \prime}(0)=2.48, x_{2,1^{\prime}, 0}^{\prime \prime}(0)=0.15, x_{2,1^{\prime}, 1}^{\prime \prime}(0)=0.00, x_{2,1^{\prime}, 2}^{\prime \prime}(0)=0.00
\end{aligned}
$$

leads to the transient solution $\boldsymbol{x}^{\prime \prime}(t)$, which can be used in 27) to approximate the response time CDF for class 1 at the DPS station. Figure F.7 shows the resulting response time CDFs at the delay and DPS stations.

We may also want to approximate the system response time CDF for the chain with respect to the delay station. The basic procedure is similar to that for approximating the response time CDF for class 1 at the DPS station. We do not demonstrate it step by step here but present two critical figures to help with understanding. Figure F.8 illustrates the service time distributions for 

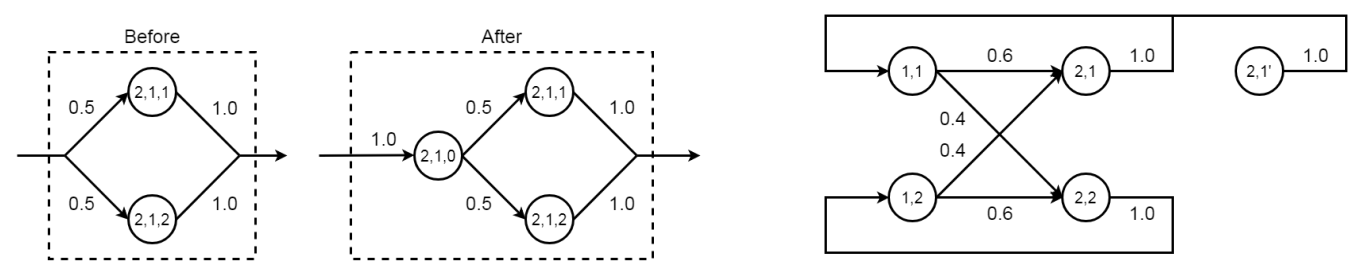

Figure F.5: The service time distribution for Figure F.6: The routing DTMC of the model class 1 at the DPS station before and after after creating an auxiliary class $1^{\prime}$ for tagging adding a start phase. class-1 jobs.

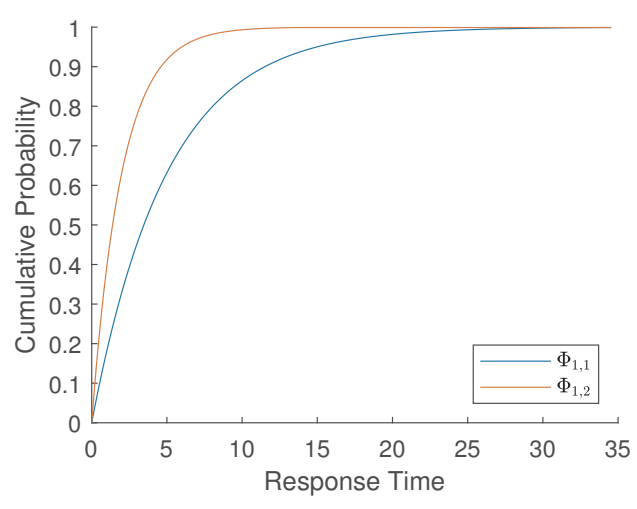

(a) Delay Station

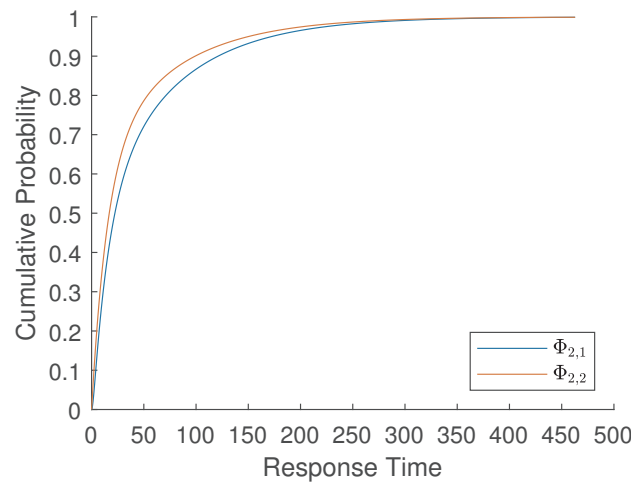

(b) DPS Station

Figure F.7: The resulting response time CDFs.

classes 1 and 2 at the delay station before and after adding end phases. Applying (20) and (21) gives the mean service times of a class- 1 and a class- 2 job at the delay station, which are $S_{1,1}=5$ and $S_{1,2}=2$ respectively. It then follows from 20 and 21 that the mean system response time of a job in the chain with respect to the delay station is $\tilde{R} p_{1-1} \approx 40.9$. With the small positive coefficient $\eta=0.03$, we set the service rate of a job in the additional phases as $\mu_{1,1,2}=\mu_{1,2,2} \approx 0.8$. Figure F.9 shows the routing DTMC of the model after creating auxiliary classes $1^{\prime}$ and $2^{\prime}$ for tagging jobs in the chain. Note that the service time distributions and the service weight for these auxiliary classes need to be identical to those of classes 1 and 2 at both delay and DPS stations. The resulting system response time CDF for the chain is shown in Figure F.10.

Finally, we demonstrate how the accuracy of the fluid approach changes with respect to the load of the DPS station. To this end, a sequence of model instances are created based on the simple example by increasing the total population of jobs from 1 to 10 . Note that the heavy load condition given by 12 is not satisfied by the first model instance. We use the fluid approach to approximate the mean queue lengths for the sequence of model instances and evaluate the errors of the results in terms of (32). As shown in Figure (F.11), the accuracy of the fluid approach increases from above 0.3 to below 0.1 as the total population of jobs grows. 

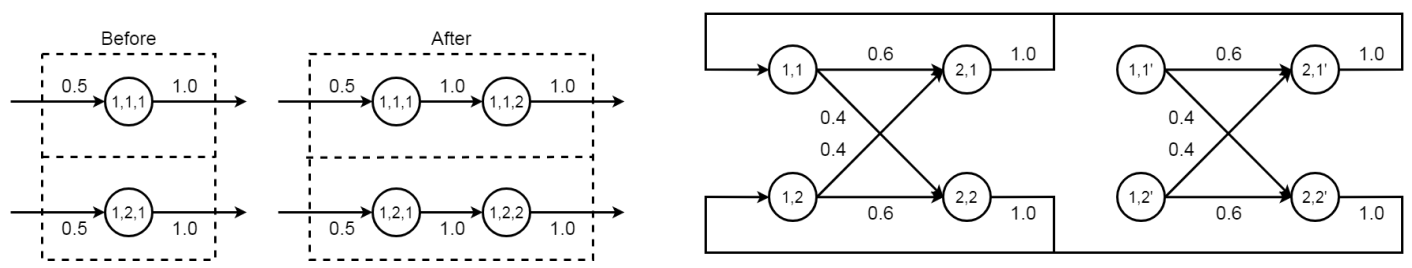

Figure F.8: The service time distributions for Figure F.9: The routing DTMC of the model classes 1 and 2 at the delay station before and after creating auxiliary classes $1^{\prime}$ and $2^{\prime}$ for tagafter adding end phases. ging jobs in the chain.

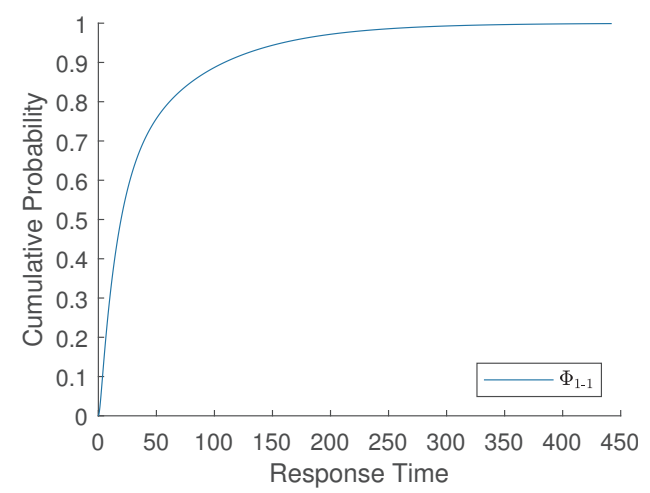

Figure F.10: The resulting system response time CDF.

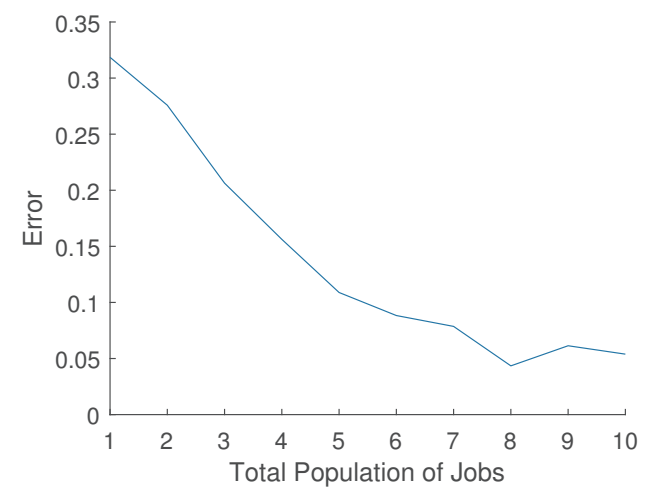

Figure F.11: The errors in using the fluid approach to analyze a sequence of model instances from light to heave load.

\section{References}

[1] A. K. Parekh, R. G. Gallager, A generalized processor sharing approach to flow control in integrated services networks: The single node case, IEEE/ACM Transactions on Networking 1 (3) (1993) 344-357.

[2] A. K. Parekh, R. G. Gallager, A generalized processor sharing approach to flow control in in- 
tegrated services networks: The multiple node case, IEEE/ACM Transactions on Networking 2 (2) (1994) 137-150.

[3] L. Kleinrock, Time-shared systems: A theoretical treatment, Journal of the ACM 14 (2) (1967) 242-261.

[4] A. Silberschatz, P. B. Galvin, Operating System Concepts, Addison-Wesley, 1998.

[5] L. Massoulié, J. W. Roberts, Bandwidth sharing and admission control for elastic traffic, Telecommunication Systems 15 (1-2) (2000) 185-201.

[6] T. Bu, D. Towsley, Fixed point approximations for TCP behavior in an AQM network, ACM SIGMETRICS Performance Evaluation Review 29 (1) (2001) 216-225.

[7] E. Altman, T. Jimenez, D. Kofman, DPS queues with stationary ergodic service times and the performance of TCP in overload, in: Proceedings of IEEE INFOCOM 2004, IEEE, 2004, pp. $975-983$.

[8] S. K. Cheung, J. L. van den Berg, R. J. Boucherie, R. Litjens, F. Roijers, An analytical packet/flow-level modelling approach for wireless LANs with quality-of-service support, in: Proceedings of the 19th International Teletraffic Congress, Beijing University of Posts and Telecommunications Press, 2005, pp. 1651-1662.

${ }_{665}[9]$ Y. Hayel, B. Tuffin, Pricing for heterogeneous services at a discriminatory processor sharing queue, in: Proceedings of NETWORKING 2005, Springer, 2005, pp. 816-827.

[10] K. Molka, G. Casale, Energy-efficient resource allocation and provisioning for in-memory database clusters, in: Proceedings of the 15th IFIP/IEEE Symposium on Integrated Network and Service Management, IEEE, 2017, pp. 19-27.

[11] K. Avrachenkov, U. Ayesta, P. Brown, R. Núñez-Queija, Discriminatory processor sharing revisited, in: Proceedings of IEEE INFOCOM 2005, IEEE, 2005, pp. 784-795.

[12] E. Altman, K. Avrachenkov, U. Ayesta, A survey on discriminatory processor sharing, Queueing Systems 53 (1-2) (2006) 53-63.

[13] S. Aalto, U. Ayesta, S. Borst, V. Misra, R. Núñez-Queija, Beyond processor sharing, ACM SIGMETRICS Performance Evaluation Review 34 (4) (2007) 36-43.

[14] F. Baskett, K. M. Chandy, R. R. Muntz, F. G. Palacios, Open, closed, and mixed networks of queues with different classes of customers, Journal of the ACM 22 (2) (1975) 248-260.

[15] J. A. Rolia, K. C. Sevcik, The method of layers, IEEE Transactions on Software Engineering 21 (8) (1995) 689-700. 
[25] S. Borst, D. van Ooteghem, B. Zwart, Tail asymptotics for discriminatory processor-sharing queues with heavy-tailed service requirements, Performance Evaluation 61 (2-3) (2005) 281298.

[26] J. Kim, B. Kim, Sojourn time distribution in the M/M/1 queue with discriminatory processor-

[27] T. Chis, P. Harrison, Higher response time moments for $\mathrm{M} / \mathrm{M} / 1$ discriminatory processor sharing queues, in: Proceedings of the 9th EAI International Conference on Performance Evaluation Methodologies and Tools, Institute for Computer Sciences, Social-Informatics and Telecommunications Engineering, 2016, pp. 145-148. 
[38] J. C. Spall, Introduction to Stochastic Search and Optimization: Estimation, Simulation, and Control, John Wiley \& Sons, 2005. processor sharing queue, ACM Transactions on Modeling and Performance Evaluation of Computing Systems 1 (1) (2016) 5.

[29] D. Mitra, A. Weiss, A closed network with a discriminatory processor-sharing server, ACM SIGMETRICS Performance Evaluation Review 17 (1) (1989) 200-208.

[30] J. A. Morrison, Asymptotic analysis of a large closed queueing network with discriminatory processor sharing, Queueing Systems 9 (1-2) (1991) 191-213.

[31] L. Bortolussi, J. Hillston, D. Latella, M. Massink, Continuous approximation of collective system behaviour: A tutorial, Performance Evaluation 70 (5) (2013) 317-349.

[32] G. Casale, M. Tribastone, P. G. Harrison, Blending randomness in closed queueing network models, Performance Evaluation 82 (C) (2014) 15-38.

[33] M. Reiser, S. S. Lavenberg, Mean-value analysis of closed multichain queuing networks, Journal of the ACM 27 (2) (1980) 313-322.

[34] E. D. Lazowska, J. Zahorjan, G. S. Graham, K. C. Sevcik, Quantitative System Performance: Computer System Analysis Using Queueing Network Models, Prentice Hall, 1984.

[35] M. Bertoli, G. Casale, G. Serazzi, JMT: Performance engineering tools for system modeling, ACM SIGMETRICS Performance Evaluation Review 36 (4) (2009) 10-15.

[36] K. M. Chandy, D. Neuse, Linearizer: A heuristic algorithm for queueing network models of computing systems, Communications of the ACM 25 (2) (1982) 126-134.

[37] W. G. Kelley, A. C. Peterson, The Theory of Differential Equations: Classical and Qualitative, Springer, 2010.

[28] A. Izagirre, U. Ayesta, I. M. Verloop, Sojourn time approximations for a discriminatory 